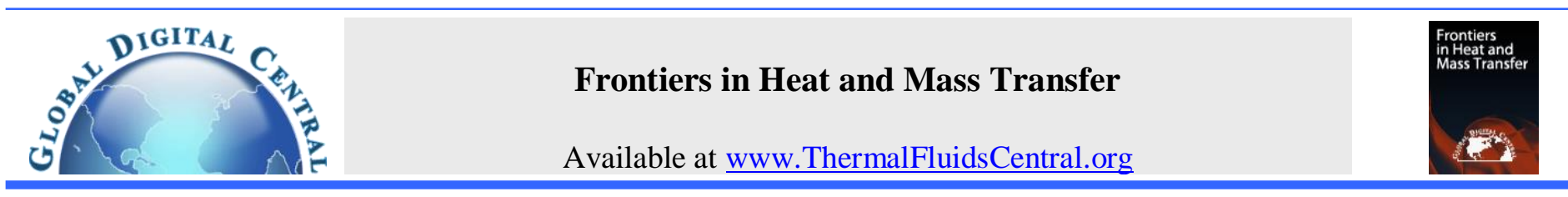

\title{
MAGNETO-CONVECTION OF ALUMINA - WATER NANOFLUID WITHIN THIN HORIZONTAL LAYERS USING THE REVISED GENERALIZED BUONGIORNO'S MODEL
}

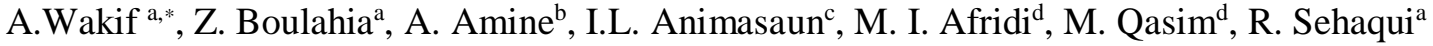 \\ ${ }^{a}$ Hassan II University, Faculty of Sciences Ä̈n Chock, Laboratory of Mechanics, B.P.5366 Mâarif, 20100 Casablanca, Morocco. \\ ${ }^{b}$ Hassan II University, Faculty of Sciences and Technology, Laboratory of Condensed Matter and Renewable Energy, Mohammedia, Morocco. \\ ${ }^{c}$ Department of Mathematical Sciences, Federal University of Technology, Akure, Nigeria. \\ ${ }^{d}$ Department of Mathematics, COMSATS University Islamabad (CUI), Park Road, Tarlai Kalan, Islamabad-455000, Pakistan.
}

\begin{abstract}
The significance of an externally applied magnetic field and an imposed negative temperature gradient on the onset of natural convection in a thin horizontal layer of alumina-water nanofluid for various sizes of spherical alumina nanoparticles (e.g., $30 \mathrm{~nm}, 35 \mathrm{~nm}, 40 \mathrm{~nm}, 45 \mathrm{~nm}$ ) and volumetric fractions (e.g., 0.01, 0.02, 0.03, 0.04) is explored and analyzed numerically in this paper. The generalized Buongiorno's mathematical model with the simplified Maxwell's equations and the Oberbeck-Boussinesq approximation were adopted to simulate the two-phase transport phenomena, in which the Brownian motion and thermophoresis aspects are taken into account. Moreover, the rheological behavior of alumina-water nanofluid and related flow are assumed to be Newtonian, incompressible and laminar. Based on the linear stability theory, the perturbed partial differential equations (PDEs) of magnetohydrodynamic convective nanofluid flow are firstly simplified formally using the normal mode analysis technique and secondly converted to a generalized eigenvalue problem considering more realistic boundary conditions, in which the thermal Rayleigh number is the associated eigenvalue. Additionally, the resulting eigenvalue problem was solved numerically using powerful collocation methods, like Chebyshev-Gauss-Lobatto Spectral Method (CGLSM) and Generalized Differential Quadrature Method (GDQM). Furthermore, the thermo-magneto-hydrodynamic stability of the nanofluidic system and the critical size of convection cells are highlighted graphically in terms of the critical thermal Rayleigh and wave numbers, for various values of the magnetic Chandrasekhar number, the volumetric fraction and the diameter of alumina nanoparticles.
\end{abstract}

Keywords: Linear Stability Analysis; Nanofluid; Alumina Nanoparticles; Magneto-Convection; Buongiorno's Model; Collocation Methods.

\section{INTRODUCTION}

In recent decades, numerous investigations have been conducted on the conventional fluids, in an attempt to enhance their intrinsic thermophysical properties and consequently improve heating and cooling systems by dispersing insoluble nano-sized particles (Maxwell, 1873) like copper, silver, gold, alumina, copper oxide, titanium dioxide, carbon nanotubes, graphene, graphene oxide and diamond into a specified fluid such as water, ethylene glycol and oil. This new type of working fluids, which was first invented by Choi (1995) is called nanofluids. In other words, the terminology of nanofluid or nanoliquid was introduced scientifically in fluid mechanics and rheology to refer to the fluids with suspended solid nanoparticles of average size less than $100 \mathrm{~nm}$. Moreover, these engineered colloidal suspensions of nanoparticles in a chosen base fluid can be synthesized under some experimental conditions to obtain stable homogeneous mixtures. Today, these new innovative materials become among the most commonly used smart fluids, which have gained the significant attention of researchers around the globe, because of their widespread applications in modern science, engineering and industry. After the pioneering theoretical work of Buongiorno (2006), other experimental studies (Buongiorno, 2009 ; Żyła et al., 2018), as well as numerical modeling investigations (Boulahia et al., 2017a, 2017b , 2017c , 2017d) have been carried out by many researchers, in order to show the excellent heat transfer performance of nanofluids and their higher energy storage capacity compared to the commonly used conventional thermal fluids. As the main result, it was found that the inclusion of a small volumetric fraction of nanoparticles inside a base fluid can increase the thermal conductivity of the mixture dramatically, and also improve its thermal performance enormously. All these thermal benefits have made the nanofluids potentially useful in many engineering applications, such as heat and mass transfer enhancement, energy efficient buildings, solar collectors, biomedical applications and many others. Due to the considerable advancement in modern technology and growing demands in the development of efficient and powerful cooling systems, the nanofluid technology has gained worldwide acceptance from researchers in recent years, mainly in the field of cooling systems like nuclear power reactors, transformer oils, devices in automotive vehicles and microelectronic components. Following the same trends, Makinde and Animasaun (2016a , 2016b), Pandey and Kumar (2016, 2017a , 2017b , 2017c), Boulahia et al. $(2016$, 2018) and Koriko et al. (2018) studied the convection heat transfer behavior of nanofluids and the significance of quartic chemical reactions through various practical engineering problems, in order to achieve a profound understanding of the importance of these liquid substances (i.e., nanofluids or nanoliquids)

*Corresponding author. Email: wakif.abderrahim@ gmail.com 
towards the advanced industrial and technological systems requiring ultra-high rates of heating or cooling.

From the chemical and physical point of views, the nanofluids can be actually synthesized in the scientific laboratories by utilizing various experimental methods. In spite of these numerous approaches, there are only two primary ways, namely single-step and two-step processes among the most widely used methods for preparing homogeneous mixtures with almost negligible agglomeration of nanoparticles. These techniques have been carried out by respecting specified experimental protocols, which require careful steps to ensure that the mixture (e.g., base fluid + nanoparticles) remains stable over a long period of time. In addition, the single-step and two-step methods have been classified according to the number of steps involved during their preparation. In the first method, the nanoparticles are prepared and dispersed simultaneously into a base fluid during the manufacturing process of nanoparticles. While in the two-step method, the nanoparticles are first produced either by physical or chemical processes, which can be thereafter dispersed into the base fluid using an intensive magnetic force agitation. In term of the economic cost, the two-step procedure is considered as the easiest method applied by the experimenters to manufacture enhanced working fluids in large and applicable scales. Regardless of the method employed for preparing nanofluids, the nanoparticles always have a tendency to agglomerate or aggregate due to the van der Waals forces. Consequently, the heavier nanoparticles tend to settle at the bottom under the impact of gravitational forces. Due to this fact, many undesirable phase instabilities driven by the agglomeration and sedimentation of nanoparticles can be induced in the medium. For this purpose, it is recommended to introduce suitable surfactants into the nanofluidic medium for suppressing these instabilities. In addition, the presence of surfactant molecules inside the nanofluid can significantly increase the repulsive forces between the nanoparticles. Physically, it is well known that when the repulsive forces are higher than the attractive van der Waals forces, the impact of the agglomeration can be quantitatively reduced for the nanoparticles.

In the light of the aforementioned results, Angayarkanni and Philip (2015) reported in their innovative work that to ensure the stability maintenance of nanofluids, it is suggested to minimize the destabilizing effect of the attractive van der Waals forces either by decreasing the size of solid nanoparticles $d_{n p}$, increasing the base fluid viscosity $\mu_{b f}$ or decreasing the density difference between the nanoparticles and the base fluid. Furthermore, it is also proved that due to the presence of the nanoparticles migration inside the base fluids, the nanofluids can achieve a substantial enhancement in the thermal transfer performances. In view of all these results, Wakif et al. (2018a) revealed that the heterogeneity of nanoparticles concentration increases locally with the temperature. The main reasons behind the non-homogeneity in the volumetric fraction of nanoparticles are the Brownian motion and thermophoresis slip mechanisms, which are evidently due to the random movements of nanoparticles and their very small dimensions. In this framework, Buongiorno (2006) in his seminal work suggested a two-component fourequation non-homogeneous equilibrium model for mass, momentum, volumetric fraction of nanoparticles and heat transfer in nanofluids by taking into account the effects of the thermophoresis phenomenon (i.e., movement of solid nanoparticles from the warmer regions to the colder regions) and Brownian motion (i.e., movement of solid nanoparticles from the greater concentration areas to those with lower concentration) as possible slip mechanisms between the base fluid and nanoparticles. In related works, Nield and Kuznetsov (2014a , 2014b) extended the validity of the Buongiorno's mathematical model for the impermeable boundaries, in which the volumetric fraction of solid nanoparticles can be passively rather than actively controlled at the boundaries. In this revised model, the nanoparticles on the impermeable walls are indirectly adjusted via the volumetric fraction gradient of nanoparticles by adopting the condition of zero nanoparticles mass flux. Physically, this newly suggested assumption for controlling the nanoparticles is more realistic as compared to other models. Likewise, this approach remains possible for the nanofluids, as long as the boundaries are assumed impermeable to the base fluid and nanoparticles. Very recently, Animasaun (2016) investigated the dynamics of water conveying alumina nanoparticles on an upper horizontal surface of paraboloid of revolution coated with three molecules of catalyst using quartic autocatalysis chemical reaction. It was observed that the horizontal velocity and temperature distribution across the flow increase with the volumetric fraction of nanoparticles. In another related study about the influence of Hall effect on the flow of $36 \mathrm{~nm}$ alumina-water and $47 \mathrm{~nm}$ alumina-water nanofluids, Animasaun et al. (2018) remarked that the maximum cross-flow velocity is attained within the fluid domain when $36 \mathrm{~nm}$ nanoparticles alumina is used.

Nowadays, the Buongiorno's two-phase approach becomes the most plausible and used model for predicting the heat transfer enhancement and analyzing the nanoparticles transportation in nanofluids, owing to its reasonable explanation. Therefore, many researchers are constantly working to develop this heterogeneous model, in order to improve its capability as an advanced mathematical model for simulating the twophase convective flows (i.e., natural, forced or mixed convection flows) and estimating the heat transfer performance of nanofluids. Lately, this model has been generalized to take care of the nanofluid flow variables, due to the effective dependence of the thermophysical properties of nanofluids on the temperature and the volume fraction of nanoparticles. Based on the revised form of the Buongiorno's two-phase model, several interesting investigations were reported by many researchers using the generalized version of the heterogeneous two-phase model. Among the more recent researches on nanofluids, it can be found a lot of related papers in the literature survey regarding the generalized Buongiorno's nanofluid model. To mention a few, Garoosi and Talebi (2017) used the Buongiorno's two - phase model to examine a numerical simulation of the combined conduction and natural convection nanofluid flows and temperature distribution in an enclosure with the presence of vertical partition or several conductive obstacles with finite thickness and thermal conductivity. In this regard, the authors used Corcione's correlations (Corcione, 2011) as the best ways for estimating the effective thermal conductivity $k_{n f}$ and dynamic viscosity $\mu_{n f}$. Alsabery et al. (2018) investigated the problem of conjugate natural convection of $\mathrm{Al}_{2} \mathrm{O}_{3}$ water nanofluids in a square cavity with concentric solid insert and isothermal corner boundaries using the non-homogenous Buongiorno's two-phase model in combination with the Corcione's correlations. Astanina et al. (2018) adopted the heterogeneous Buongiorno's model to investigate the natural convection in a partially heated enclosure by considering the dependence of thermophysical properties of $\mathrm{CuO}$-water nanofluids on temperature $T^{*}$ and volume fraction $\phi^{*}$. Accordingly, the researchers who authored this work recommended employing Chon's correlation (Chon et al., 2005) for predicting the effective thermal conductivity $k_{n f}$ and another powerful model developed by Nguyen et al. (2007) to estimate the effective dynamic viscosity $\mu_{n f}$ based on experimental data.

Owing to the relevance of heat transfer of nanofluids and their widespread usage area in today's advanced nanotechnologies, other related investigations on $\mathrm{SiO}_{2}$ - ethylene glycol nanofluids have been carried out experimentally by Żyła and Fal (2017) to measure the effective dynamic viscosity $\mu_{n f}$ and thermal conductivity $k_{n f}$ as well as the electrical conductivity $\sigma_{n f}$. This great work has been subsequently extended and analyzed by Żyła et al. (2018) in the same manner for ethylene glycol $(E G)$ - based nanofluids containing nanodiamonds $(N D)$, in order to explore the rheological, thermophysical and dielectric properties of these nanofluids. Among the main results of these experimental investigations, it was found that the thermal conductivity $k_{n f}$ and the electrical conductivity $\sigma_{n f}$ of $\mathrm{SiO}_{2}$ - ethylene glycol and $N D$ - ethylene glycol nanofluids generally increase with the volumetric fraction of solid nanoparticles $\phi_{0}$ according to the specified empirical correlations. Similarly, it was furtherly reported that the electrical conductivity $\sigma_{n f}$ can be increased with the inclusion of some typical 
nanoparticles within a specified base fluid. For instance, Naddaf and Heris, (2018) measured the electrical conductivity $\sigma_{n f}$ of diesel oil $(D O)$ - based nanofluids containing graphene nanoplatelets $(G N P)$ or multi-walled carbon nanotubes (MWCNT) as solid nanoparticles and hexylamine $(H A)$ or oleic acid $(O A)$ as suitable surfactants. In this investigation, the electrical conductivity was greatly improved with the increase in nanomaterials concentration and temperature. This stems from the fact that the interaction between the nanoparticles is increased with the concentration as well as the electrons of the solid phase can be thermally excited from the valence band to the conduction band for enhancing the electrical conductance of the mixture and producing the so-called electrically conducting nanofluid, whose the flow behavior can be influenced by the existence of Lorentz forces generated within the medium under the effect of an externally applied magnetic field.

Over the last several decades, a wide variety of practical engineering problems dealing with the magnetohydrodynamic (MHD) convective heat and mass transfer flows have attracted substantial attention by many researchers, due to their widespread and vital potential applications in technological and industrial disciplines. More recently, tremendous research works focusing on the magnetohydrodynamic convective flows of electrically conducting fluids or nanofluids were reported previously by many researchers. Later, Amanulla et al. (2018) performed a thorough numerical investigation to simulate the steady MHD convective flow of Carreau non-Newtonian fluid past an isothermal sphere by applying Keller-Box Method (KBM). Qasim et al. (2018) conducted an innovative numerical simulation of MHD peristaltic flow with variable electrical conductivity and joule dissipation by utilizing Generalized Differential Quadrature Method (GDQM). On the other hand, a comprehensive survey was done by Wakif et al. (2017a) on the thermo-magneto-hydrodynamic stability of nanofluids saturating porous mediums by means of Chebyshev-Gauss-Lobatto Spectral Method (CGLSM). Similarly, Rana et al. (2017) studied the onset of thermomagneto-hydrodynamic instability in a rotating viscoelastic nanofluid layer under the influence of an internal heat source with the help of Galerkin Weighted Residuals Technique (GWRT). Also, Akbarzadeh (2018) carried out a numerical study concerning the effect of a purely internal heat generation and chemical reaction on the onset of thermomagneto-hydrodynamic instabilities inside a porous medium saturated by a nanofluid by using GWRT. In another stability problem, Wakif et al. (2018b) proposed a generalized mathematical formulation to perform numerical examinations of the thermo-magneto-hydrodynamic stability of some metallic nanofluids by considering the Buongiorno's and Corcione's models and utilizing Runge-Kutta-Fehlberg Method (RKFM) as a powerful approach for implementing the stability problems.

Based on the above-cited papers concerning the thermo-magnetohydrodynamic stability of nanofluids, it is found that there is a great lack of numerical works dealing with the realistic analysis of the thermomagneto-hydrodynamic instabilities occurred inside the electrically conducting nanofluids under the effects of buoyancy and Lorentz forces. However, to the best of author's knowledge, the revised form of the generalized Buongiorno's two-phase model with the assumption of zero nanoparticles mass flux condition has not been given any attention from researchers, despite the importance of magneto-convection in many thermal engineering systems. Inspired by the above-mentioned works, our configuration of interest focuses on using the revised Buongiorno's mathematical model as a more realistic approach for analyzing the thermo-magneto-hydrodynamic stability of alumina-water nanofluids by considering the Chon's and Corcione's nanofluid models for estimating the effective thermal conductivity $k_{n f}$ and dynamic viscosity $\mu_{n f}$, respectively. Likewise, the resulting stability equations are solved numerically with a good accuracy by utilizing Chebyshev-Gauss-Lobatto Spectral Method (CGLSM) and Generalized Differential Quadrature Method (GDQM), for non-slip, impermeable, zero nanoparticles mass flux and isothermal boundary conditions. Moreover, the present findings are multiply validated by comparing our numerical outcomes with the results of the existing literature. Furthermore, the effects of various emerging parameters (i.e., $Q, \phi_{0}$ and $d_{n p}$ ) on the criterion for the onset of magneto-convection in alumina-water nanofluids are provided in the form of graphical and tabular illustrations coupled with comprehensive discussions.

\section{PROBLEM FORMULATION}

The schematic diagram of the studied problem is depicted in Fig. 1. In this physical configuration, we consider a thin horizontal layer of a dilute colloidal suspension containing spherical alumina nanoparticles $\mathrm{Al}_{2} \mathrm{O}_{3}$ dispersed into a pure water $\mathrm{H}_{2} \mathrm{O}$. A fixed spatial Cartesian frame of reference $\left(x^{*}, y^{*}, z^{*}\right)$ is located in the lower level of the plates (IHP) and (ICP), in which the $z^{*}$-axis is directed vertically upwards. Also, the studied homogeneous mixture $\left(\mathrm{Al}_{2} \mathrm{O}_{3}+\mathrm{H}_{2} \mathrm{O}\right)$ is assumed electrically conducting nanofluid, incompressible fluid, has Newtonian rheological behavior, confined between two electrically non-conducting infinite parallel plates, subjected to a uniform gravitational field $\mathbf{g}$, heated from below and affected by an external transverse magnetic field $\mathbf{H}_{\mathbf{0}}$ with a constant magnetic strength $H_{0}$. In order to simplify the proposed model, the viscous dissipation, Ohmic heating and Hall effects are not taken into account in the present numerical examination. As imposed boundary conditions, the lower and upper boundaries are maintained at constant temperatures $T_{h}$ and $T_{c}\left(T_{c}<T_{h}\right)$, respectively, whereas the volumetric fraction of solid nanoparticles is controlled passively at the horizontal impermeable plates, in such a way that the vertical component $J_{Z}^{*}$ of the nanoparticles mass flux is assumed to be zero on these boundaries (i. e., $\left.\left(\partial \phi^{*} / \partial z^{*}\right)+\left[D_{T} /\left(D_{B} T_{c}\right)\right]\left(\partial T^{*} / \partial z^{*}\right)=0\right)$, in which there are no slip velocities between the nanofluid and the plates (IHP) and (ICP). For a given volumetric fraction of nanoparticles $\phi_{0}$, the thermophysical properties of alumina-water nanofluids (e.g., dynamic viscosity $\mu_{n f}$, thermal conductivity $k_{n f}$, specific heat $c_{n f}$, magnetic permeability $\bar{\mu}_{n f}$ and electrical conductivity $\sigma_{n f}$ ) are assumed to remain constant in the vicinity of the cold plate temperature $T_{\mathrm{c}}$, except for the density $\rho$ in the gravitational force $\mathbf{F}_{\mathbf{g}}=-\rho g \mathbf{e}_{\mathbf{z}}$, which is estimated formally based on the Oberbeck-Boussinesq approximation.

According to Buongiorno (2006) and Wakif et al. (2017b , 2018b), the various partial differential equations governing the nanofluid flow, heat and mass transfer in the presence of an external magnetic field are written as follows:

$$
\begin{aligned}
& \nabla^{*} \cdot \mathbf{V}^{*}=0 \\
& \rho_{n f}\left[\frac{\partial \mathbf{V}^{*}}{\partial t^{*}}+\left(\mathbf{V}^{*} \cdot \nabla^{*}\right) \mathbf{V}^{*}\right]=-\nabla^{*} P^{*}+\mu_{n f} \nabla^{* 2} \mathbf{V}^{*}+\mathbf{F}_{\mathbf{g}}+\mathbf{F}_{\mathbf{m}} \\
& \frac{\partial T^{*}}{\partial t^{*}}+\left(\mathbf{V}^{*} \cdot \nabla^{*}\right) T^{*}=\frac{k_{n f}}{(\rho c)_{n f}} \nabla^{* 2} T^{*}+\frac{(\rho c)_{n p}}{(\rho c)_{n f}}\left[\begin{array}{c}
D_{B} \nabla^{*} \phi^{*} \cdot \nabla^{*} T^{*} \\
+\frac{D_{T}}{T_{c}} \nabla^{*} T^{*} \cdot \nabla^{*} T^{*}
\end{array}\right] \\
& \frac{\partial \phi^{*}}{\partial t^{*}}+\left(\mathbf{V}^{*} \cdot \nabla^{*}\right) \phi^{*}=D_{B} \nabla^{* 2} \phi^{*}+\frac{D_{T}}{T_{c}} \nabla^{* 2} T^{*} \\
& \frac{\partial \mathbf{H}^{*}}{\partial t^{*}}+\left(\mathbf{V}^{*} . \nabla^{*}\right) \mathbf{H}^{*}=\left(\mathbf{H}^{*} . \nabla^{*}\right) \mathbf{V}^{*}+\eta_{n f} \nabla^{* 2} \mathbf{H}^{*} \\
& \nabla^{*} . \mathbf{H}^{*}=0
\end{aligned}
$$

Within the framework of the above-mentioned assumptions, the gravitational body force $\mathbf{F}_{\mathbf{g}}$ (Wakif et al., 2018a , 2018b, 2018c) and the Lorentz force $\mathbf{F}_{\mathbf{m}}$ (Chandrasekhar, 1961) are expressed by:

$$
\begin{gathered}
\mathbf{F}_{\mathbf{g}}=-\left[1-\beta_{n f}\left(T^{*}-T_{c}\right)+\beta_{\phi}\left(\phi^{*}-\phi_{0}\right)\right] \rho_{n f} g \mathbf{e}_{\mathbf{z}} \\
\mathbf{F}_{\mathbf{m}}=\frac{\bar{\mu}_{n f}}{4 \pi}\left(\nabla^{*} \times \mathbf{H}^{*}\right) \times \mathbf{H}^{*}
\end{gathered}
$$




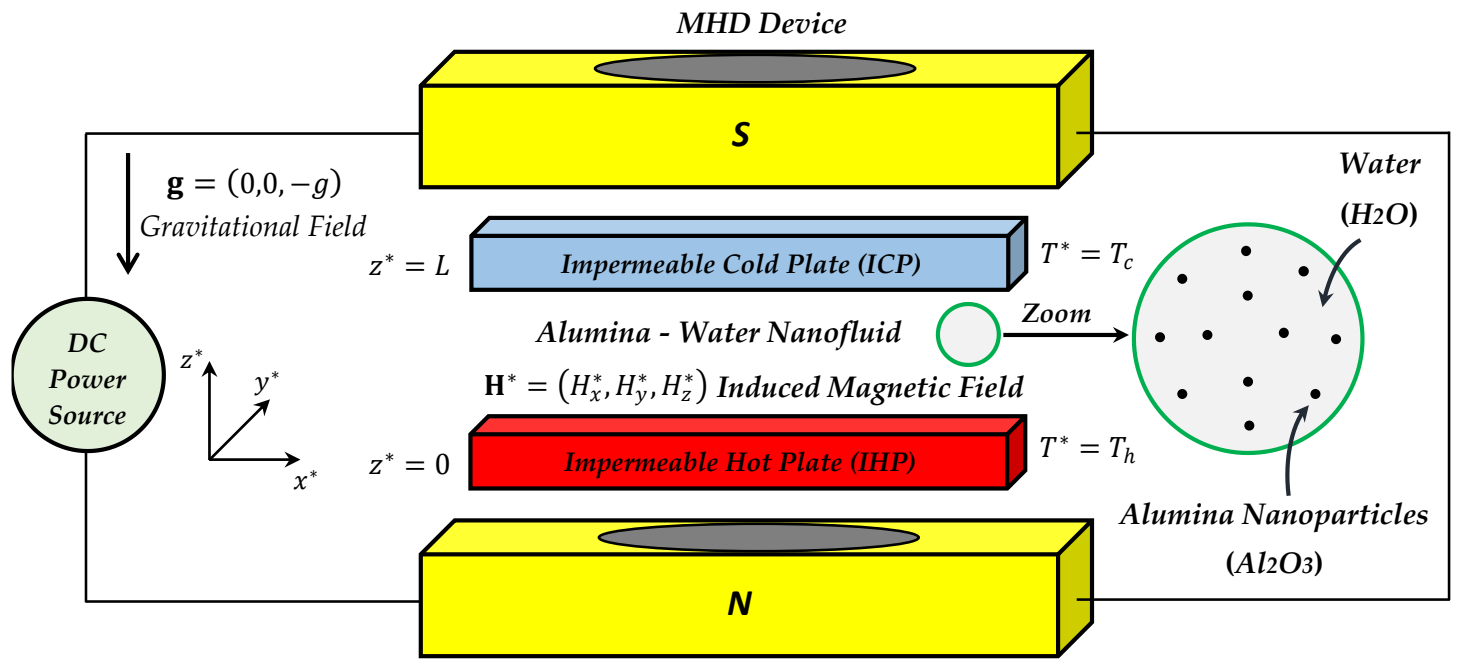

Fig. 1 Description of the physical flow model.

Here, $\mathbf{V}^{*}$ is the nanofluid velocity vector, $\mathbf{H}^{*}$ denotes the induced magnetic field and $\nabla^{*}$ refers to the nabla vector operator, whereas $t^{*}, P^{*}$, $T^{*}, \phi^{*}$ represent the time, the pressure, the temperature and the volume fraction of solid nanoparticles, respectively, where $\mathbf{V}^{*}=\left(u^{*}, v^{*}, w^{*}\right)$, $\mathbf{H}^{*}=\left(H_{x}^{*}, H_{y}^{*}, H_{z}^{*}\right)$ and $\nabla^{*}=\left(\partial / \partial x^{*}, \partial / \partial y^{*}, \partial / \partial z^{*}\right)$.

Following Buongiorno (2006), McNab and Meisen (1973), the Brownian diffusion coefficient $D_{B}$ and the thermophoretic diffusion coefficient $D_{T}$ shown in Eqs. (3) and (4) can be calculated at the reference state (i. e.,$\left.\left(T^{*}, \phi^{*}\right)=\left(T_{c}, \phi_{0}\right)\right)$ by:

$$
\begin{gathered}
D_{B}=\frac{k_{B} T_{c}}{3 \pi \mu_{b f} d_{n p}} \\
D_{T}=0.26\left(\frac{k_{b f}}{2 k_{b f}+k_{n p}}\right)\left(\frac{\mu_{b f}}{\rho_{b f}}\right) \phi_{0}
\end{gathered}
$$

In addition, the physical problem under consideration is subjected to the following boundary conditions:

$$
\begin{aligned}
& w^{*}=\frac{\partial w^{*}}{\partial z^{*}}=0, T^{*}=T_{h}, \frac{\partial \phi^{*}}{\partial z^{*}}+\frac{D_{T}}{D_{B} T_{c}} \frac{\partial T^{*}}{\partial z^{*}}=0 \text { at } z^{*}=0 \\
& w^{*}=\frac{\partial w^{*}}{\partial z^{*}}=0, T^{*}=T_{c}, \frac{\partial \phi^{*}}{\partial z^{*}}+\frac{D_{T}}{D_{B} T_{c}} \frac{\partial T^{*}}{\partial z^{*}}=0 \text { at } z^{*}=L
\end{aligned}
$$

As is well-known, the nanofluid properties depend strongly on the values of temperature and volumetric fraction of nanoparticles taken into account during the estimation. Accordingly, the effective thermophysical properties of Alumina - water nanofluids like the density $\rho_{n f}$, the thermal diffusivity $\alpha_{n f}$, the specific heat capacity $(\rho c)_{n f}$, the volumetric mass expansion coefficient $(\rho \beta)_{n f}$, the electrical conductivity $\sigma_{n f}$, the magnetic permeability $\bar{\mu}_{n f}$ and the magnetic diffusivity $\eta_{n f}$ are given by Garnett (1905), Sihvola and Lindell (1992) and Wakif et al. (2018a , 2018b) as follows:

$$
\begin{gathered}
\rho_{n f}=\left(1-\phi_{0}\right) \rho_{b f}+\phi_{0} \rho_{n p} \\
\alpha_{n f}=\frac{k_{n f}}{(\rho c)_{n f}} \\
(\rho c)_{n f}=\left(1-\phi_{0}\right)(\rho c)_{b f}+\phi_{0}(\rho c)_{n p} \\
(\rho \beta)_{n f}=\left(1-\phi_{0}\right)(\rho \beta)_{b f}+\phi_{0}(\rho \beta)_{n p} \\
\frac{\sigma_{n f}}{\sigma_{b f}}=1+\frac{3\left[\left(\sigma_{n p} / \sigma_{b f}\right)-1\right] \phi_{0}}{\left[\left(\sigma_{n p} / \sigma_{b f}\right)+2\right]-\left[\left(\sigma_{n p} / \sigma_{b f}\right)-1\right] \phi_{0}}
\end{gathered}
$$

$$
\begin{gathered}
\frac{\bar{\mu}_{n f}}{\bar{\mu}_{b f}}=1+\frac{3\left[\left(\bar{\mu}_{n p} / \bar{\mu}_{b f}\right)-1\right] \phi_{0}}{\left[\left(\bar{\mu}_{n p} / \bar{\mu}_{b f}\right)+2\right]-\left[\left(\bar{\mu}_{n p} / \bar{\mu}_{b f}\right)-1\right] \phi_{0}} \\
\eta_{n f}=\frac{1}{4 \pi \bar{\mu}_{n f} \sigma_{n f}}
\end{gathered}
$$

Here, the subscripts $n f, n p$ and $b f$ represents the nanofluid, the solid nanoparticles and the base fluid, respectively.

Furthermore, the magnetic permeabilities of alumina $\bar{\mu}_{n p}$ and water $\bar{\mu}_{b f}$ can be concluded from their corresponding magnetic susceptibilities $\chi_{n p}$ and $\chi_{b f}$, respectively, using the following formulas:

$$
\begin{aligned}
& \bar{\mu}_{n p}=\bar{\mu}_{0}\left(1+\chi_{n p}\right) \\
& \bar{\mu}_{b f}=\bar{\mu}_{0}\left(1+\chi_{b f}\right)
\end{aligned}
$$

Here, $\bar{\mu}_{0}$ represents the magnetic permeability of free space, where $\bar{\mu}_{0}=4 \pi \times 10^{-7} \mathrm{H} \mathrm{m}^{-1}$. In order to perform a realistic prediction of the other effective thermophysical properties of $\mathrm{Al}_{2} \mathrm{O}_{3}$ - water nanofluids, the effective thermal conductivity $k_{n f}$ and dynamic viscosity $\mu_{n f}$ can be computed efficiently at the reference state by making use of Chon's and Corcione's nanofluid models, respectively, which are expressed by :

$$
\begin{gathered}
\frac{k_{n f}}{k_{b f}}=1+64.7 \phi_{0}^{0.746}\left(\frac{d_{b f}}{d_{n p}}\right)^{0.369}\left(\frac{k_{n p}}{k_{b f}}\right)^{0.7476} \operatorname{Pr}^{0.9955} \operatorname{Re}^{1.2321} \\
\frac{\mu_{n f}}{\mu_{b f}}=\frac{1}{1-34.87\left(d_{n p} / d_{b f}\right)^{-0.3} \phi_{0}^{1.03}}
\end{gathered}
$$

The Reynolds number $R_{e}$ and the molecular diameter of water $d_{b f}$ shown above can be computed from:

$$
\begin{gathered}
R_{e}=\frac{\rho_{b f} k_{B} T_{\mathrm{c}}}{3 \pi \mu_{b f}{ }^{2} l_{b f}} \\
d_{b f}=0.1\left(\frac{6 M}{N_{a v} \pi \rho_{f 0}}\right)^{\frac{1}{3}}
\end{gathered}
$$

Here, $k_{B}$ represents the Boltzmann constant, $l_{b f}$ denotes the meanfree path for water and $d_{n p}$ refers to the diameter of alumina nanoparticles $\mathrm{Al}_{2} \mathrm{O}_{3}$, whereas $\mathrm{M}, \mathrm{N}_{a v}$ and $\rho_{f 0}$ show the molecular mass weight of water, the Avogadro number and the density of water at $293 \mathrm{~K}$, respectively, where $k_{B}=1.38066 \times 10^{-23} \mathrm{JK}^{-1}, l_{b f}=0.17 \mathrm{~nm}$, $M=18 \mathrm{~g} \mathrm{~mol}^{-1}, N_{a v}=6.022 \times 10^{23} \mathrm{~mol}^{-1}$ and $\rho_{f 0}=998 \mathrm{~kg} \mathrm{~m}^{-3}$. 
Consequently, by virtue of Eqs. (13) - (25), the thermophysical properties $\rho_{n f}, \alpha_{n f},(\rho c)_{n f},(\rho \beta)_{n f}, \sigma_{n f}, \bar{\mu}_{n f}, \eta_{n f}, k_{n f}$ and $\mu_{n f}$ of $\mathrm{Al}_{2} \mathrm{O}_{3}$ - water nanofluids can be computed realistically based on the thermophysical properties of alumina nanoparticles $\mathrm{Al}_{2} \mathrm{O}_{3}$ and pure water $\mathrm{H}_{2} \mathrm{O}$ given by Schenck (1996) and Mehmood et al. (2017) at $T_{c}=300 \mathrm{~K}$ as shown in Table 1 .

Table 1 Thermophysical properties of water and alumina nanoparticles.

\begin{tabular}{|c|c|c|}
\hline \multirow{2}{*}{ Properties } & Pure Water & Alumina Nanoparticles \\
\hline & $\mathrm{H}_{2} \mathrm{O}$ & $\mathrm{Al}_{2} \mathrm{O}_{3}$ \\
\hline$d\left(\times 10^{-9} \mathrm{~m}\right)$ & 0.385 & $30-45$ \\
\hline$\rho\left(k g m^{-3}\right)$ & 997.1 & 3970 \\
\hline$c\left(J k g^{-1} K^{-1}\right)$ & 4179 & 765 \\
\hline$k\left(W m^{-1} K^{-1}\right)$ & 0.613 & 40 \\
\hline$\beta\left(\times 10^{-5} K^{-1}\right)$ & 21 & 0.85 \\
\hline$\sigma\left(S m^{-1}\right)$ & 0.05 & $10^{-10}$ \\
\hline$\chi\left(\times 10^{-6}\right)$ & -9.05 & -18.1 \\
\hline$\mu\left(\times 10^{-5} \mathrm{Pas}\right)$ & 89 & --------- \\
\hline
\end{tabular}

In order to reformulate the present physical problem in another way, the governing partial differential equations can be written in nondimensional form by introducing the following dimensionless variables:

$$
\begin{gathered}
(x, y, z)=\left(x^{*}, y^{*}, z^{*}\right) / L, t=\left(\alpha_{b f} / L^{2}\right) t^{*}, \\
P=\left[L^{2} /\left(\rho_{b f} \alpha_{b f}^{2}\right)\right] P^{*}, \mathrm{~T}=\left(T^{*}-T_{c}\right) / \Delta T, \\
\phi=\left(\phi^{*}-\phi_{0}\right) / \phi_{0}, \mathbf{V}=\left(L / \alpha_{b f}\right) \mathbf{V}^{*}, \quad \mathbf{H}=\mathbf{H}^{*} / H_{0} .
\end{gathered}
$$

Then, after making use of the non-dimensionalization procedure, Eqs. (1) - (6) reduce to:

$$
\begin{gathered}
\nabla . \mathbf{V}=0 \\
\frac{\partial \mathbf{V}}{\partial \mathrm{t}}+(\mathbf{V} \cdot \nabla) \mathbf{V}=-\frac{1}{f_{\rho}} \nabla p+f_{1} \nabla^{2} \mathbf{V}+\left[\begin{array}{c}
\left(f_{2} R_{a} T-f_{3} \phi\right) \mathbf{e}_{\mathbf{z}} \\
+f_{4}(\nabla \times \mathbf{H}) \times \mathbf{H}
\end{array}\right] \\
\frac{\partial T}{\partial t}+(\mathbf{V} . \nabla) T=f_{5} \nabla^{2} T+f_{6} \nabla \phi \cdot \nabla T+f_{7} \nabla T . \nabla T \\
\frac{\partial \phi}{\partial t}+(\mathbf{V} \cdot \nabla) \phi=f_{8} \nabla^{2} \phi+f_{9} \nabla^{2} T \\
\frac{\partial \mathbf{H}}{\partial t}+(\mathbf{V} \cdot \nabla) \mathbf{H}=(\mathbf{H} \cdot \nabla) \mathbf{V}+f_{10} \nabla^{2} \mathbf{H} \\
\nabla . \mathbf{H}=0
\end{gathered}
$$

Here, the modified dimensionless pressure $p$ shown in Eq. (28) is given by $p=P+f_{\rho} P_{r} R_{a} z /\left(\beta_{b f} \Delta T\right)$, where $\Delta T=T_{h}-T_{c}$.

In view of Eq. (26), the boundary conditions (11) and (12) become:

$$
\begin{aligned}
& w=\frac{\partial w}{\partial z}=0, T=1, \frac{\partial \phi}{\partial z}=-\left(\frac{N_{t}}{N_{b}}\right) \frac{\partial T}{\partial z} \text { at } z=0 \\
& w=\frac{\partial w}{\partial z}=0, T=0, \frac{\partial \phi}{\partial z}=-\left(\frac{N_{t}}{N_{b}}\right) \frac{\partial T}{\partial z} \text { at } z=1
\end{aligned}
$$

As mentioned before, the set $\left\{f_{i} / 1 \leq i \leq 10\right\}$ of simplifying coefficients used in Eqs. (28) - (31) are given by:

$$
\begin{gathered}
f_{1}=\left(\frac{f_{\mu}}{f_{\rho}}\right) P_{r}, f_{2}=\left(\frac{f_{(\rho \beta)}}{f_{\rho}}\right) P_{r}, f_{3}=\left(\frac{1}{f_{\rho}}\right) R_{N} P_{r}, \\
f_{4}=\left(\frac{f_{\bar{\mu}}}{f_{\rho}}\right) \frac{P_{r}^{2} Q}{P_{r M}}, f_{5}=\frac{f_{k}}{f_{(\rho c)}}, f_{6}=\left(\frac{1}{f_{(\rho c)}}\right) N_{b}, \\
f_{7}=\left(\frac{1}{f_{(\rho c)}}\right) N_{t}, f_{8}=\frac{1}{L_{e}}, f_{9}=\frac{N_{t}}{N_{b} L_{e}}, f_{10}=\left(\frac{1}{f_{\bar{\mu}} f_{\sigma}}\right) \frac{P_{r}}{P_{r M}} .
\end{gathered}
$$

Additionally, the relative thermophysical properties $f_{\rho}, f_{\mu}, f_{\bar{\mu}}, f_{k}$, $f_{\sigma}, f_{(\rho \beta)}$ and $f_{(\rho c)}$ shown above are given by:

$$
\begin{aligned}
& f_{\rho}=\frac{\rho_{n f}}{\rho_{b f}}, f_{\mu}=\frac{\mu_{n f}}{\mu_{b f}}, f_{\bar{\mu}}=\frac{\bar{\mu}_{n f}}{\bar{\mu}_{b f}}, f_{k}=\frac{k_{n f}}{k_{b f}}, \\
& f_{\sigma}=\frac{\sigma_{n f}}{\sigma_{b f}}, f_{(\rho \beta)}=\frac{(\rho \beta)_{n f}}{(\rho \beta)_{b f}}, f_{(\rho c)}=\frac{(\rho c)_{n f}}{(\rho c)_{b f}} .
\end{aligned}
$$

The non-dimensional parameters $R_{a}, R_{N}, Q, N_{b}, N_{t}, L_{e}, P_{r}$ and $P_{r M}$ appeared in Eq. (35) represents the thermal Rayleigh number, the concentration Rayleigh number, the magnetic Chandrasekhar number, the Brownian motion parameter, the thermophoresis parameter, the Lewis number, the Prandtl number and the Magnetic Prandtl number, respectively. These non-dimensional physical quantities can be regarded as the pertinent parameters of the present stability problem, which are defined as follows:

$$
\begin{gathered}
R_{a}=\frac{(\rho \beta)_{b f} \Delta T g L^{3}}{\mu_{b f} \alpha_{b f}}, R_{N}=\frac{\left(\rho_{n p}-\rho_{b f}\right) g L^{3} \phi_{0}}{\mu_{b f} \alpha_{b f}} \\
Q=\frac{\bar{\mu}_{b f} L^{2} H_{0}^{2}}{4 \pi \eta_{b f} \mu_{b f}}, N_{b}=\frac{D_{B}(\rho c)_{n p} \phi_{0}}{\alpha_{b f}(\rho c)_{b f}}, N_{t}=\frac{D_{T}(\rho c)_{n p} \Delta T}{\alpha_{b f}(\rho c)_{b f} T_{c}} \\
L_{e}=\frac{\alpha_{b f}}{D_{B}}, P_{r}=\frac{\mu_{b f}}{\rho_{b f} \alpha_{b f}}, P_{r M}=\frac{\mu_{b f}}{\rho_{b f} \eta_{b f}} .
\end{gathered}
$$

Based on the aforementioned nanofluid models proposed for $\mathrm{Al}_{2} \mathrm{O}_{3}$ water nanofluids, the relative thermophysical properties $f_{\rho}, f_{\mu}, f_{\bar{\mu}}, f_{k}$, $f_{\sigma}, f_{(\rho \beta)}$ and $f_{(\rho c)}$ in combination with the physical parameters $R_{N}, L_{e}$, $N_{b}$ and $N_{t}$ are accurately computed from Eqs. (36) and (37) and then summarized in Table 2 and Table 3, for varying values of $d_{n p}$ and $\phi_{0}$. Here, these physical quantities are obtained by considering fixed values for the layer depth $L$, the reference temperature $T_{c}$ and the temperature difference $\Delta T$, where $0.01 \leq \phi_{0} \leq 0.04,30 \mathrm{~nm} \leq d_{n p} \leq 45 \mathrm{~nm}$, $L=4.1 \times 10^{-5} \mathrm{~m}, T_{c}=300 \mathrm{~K}$ and $\Delta T=1 \mathrm{~K}$.

\subsection{Basic Solutions}

The thermo-magneto-hydrodynamic stability problem described by Eqs. (27) - (32) and the boundary conditions (33) and (34) has a steady state solution. In this particular state, the nanofluid is assumed to be at rest (i.e., $\left.\mathbf{V}_{\mathbf{b}}=(0,0,0)\right)$, in which the pressure $P_{b}$, the temperature $T_{b}$, the volumetric fraction of nanoparticles $\phi_{b}$ as well as the components $\left(H_{x b}, H_{y b}, H_{z b}\right)$ of the magnetic field $\mathbf{H}_{\mathbf{b}}$ depend solely on $z$. Hence, under these restricted considerations, the basic state of the nanofluidic system is defined by:

$$
\begin{gathered}
\frac{d P_{b}}{d z}=f_{2} f_{\rho} R_{a} T_{b}-f_{3} f_{\rho} \phi_{b}-\frac{f_{\rho} P_{r} R_{a}}{\beta_{b f} \Delta T} \\
T_{b}=1-z \\
\phi_{b}=N_{A}\left(z-\frac{1}{2}\right) \\
\left(H_{x b}, H_{y b}, H_{z b}\right)=(0,0,1)
\end{gathered}
$$

In addition to the above findings, it is important to mention here that the basic volumetric fraction $\phi_{b}$ of solid nanoparticles established in Eq. (40) is found analytically by taking into account the newest wakif's results (Wakif et al., 2016, 2018a , 2018b , 2018c) concerning the revised nanofluid model, such that:

$$
\begin{gathered}
\bar{\phi}_{b}^{*}=\frac{1}{L} \int_{0}^{L} \phi_{b}^{*} d z^{*}=\phi_{0} \\
\int_{0}^{1} \phi_{b} d z=0
\end{gathered}
$$


Here, $\bar{\phi}_{b}^{*}$ is the mean value of the dimensional volumetric fraction of nanoparticles $\phi_{b}^{*}$.

\subsection{Perturbation Equations}

In order to examine the linear stability of the basic state defined previously by Eqs. (38) - (41), it is more convenient to superimpose infinitesimal perturbations $\mathbf{V}^{\prime}, P^{\prime}, T^{\prime}, \phi^{\prime}$ and $\mathbf{H}^{\prime}$ on the basic solutions $\mathbf{V}_{\mathbf{b}}, P_{b}, T_{b}, \phi_{b}$ and $\mathbf{H}_{\mathbf{b}}$, respectively, in such a way that:

$\mathbf{V}=\mathbf{V}^{\prime}, P=P_{b}+P^{\prime}, T=T_{b}+T^{\prime}, \phi=\phi_{b}+\phi^{\prime}, \mathbf{H}=\mathbf{e}_{\mathbf{z}}+\mathbf{H}^{\prime}$.

Hence, after substituting the above expressions into Eqs. (27) - (32) and neglecting the nonlinear terms arising in the resulting differential system, we obtain the following linearized perturbation equations:

$$
\begin{gathered}
\nabla \cdot \mathbf{V}^{\prime}=0 \\
\frac{\partial \mathbf{V}^{\prime}}{\partial t}=-\frac{1}{f_{\rho}} \nabla P^{\prime}+f_{1} \nabla^{2} \mathbf{V}^{\prime}+\left[\begin{array}{c}
\left(f_{2} R_{a} T^{\prime}-f_{3} \phi^{\prime}\right) \mathbf{e}_{\mathbf{z}} \\
+f_{4}\left(\nabla \times \mathbf{H}^{\prime}\right) \times \mathbf{e}_{\mathbf{z}}
\end{array}\right] \\
\frac{\partial T^{\prime}}{\partial t}-w^{\prime}=f_{5} \nabla^{2} T^{\prime}-\left(f_{6} \frac{\partial \phi^{\prime}}{\partial z}+f_{7} \frac{\partial T^{\prime}}{\partial z}\right) \\
\frac{\partial \phi^{\prime}}{\partial t}+\frac{N_{t}}{N_{b}} w^{\prime}=f_{8} \nabla^{2} \phi^{\prime}+f_{9} \nabla^{2} T^{\prime} \\
\frac{\partial \mathbf{H}^{\prime}}{\partial t}=\frac{\partial \mathbf{V}^{\prime}}{\partial z}+f_{10} \nabla^{2} \mathbf{H}^{\prime} \\
\nabla \cdot \mathbf{H}^{\prime}=0
\end{gathered}
$$

For the resulting equations (45) - (50), the corresponding boundary conditions are stated as:

$$
w^{\prime}=\frac{\partial w^{\prime}}{\partial z}=T^{\prime}=\frac{\partial \phi^{\prime}}{\partial z}+\frac{N_{t}}{N_{b}} \frac{\partial T^{\prime}}{\partial z}=0 \quad \text { at } \quad z=0,1
$$

By operating curl twice on Eq. (46), the pressure term $P^{\prime}$ can be assuredly removed from this equation. Hence, after some simplifications that take into account Eqs. (45) and (50), the $z$-components of Eqs. (46) and (49) can be formulated as follows:

$$
\begin{gathered}
\frac{\partial}{\partial t}\left(\nabla^{2} w^{\prime}\right)=f_{1} \nabla^{4} w^{\prime}+f_{2} R_{a} \nabla_{2}^{2} T^{\prime}-f_{3} \nabla_{2}^{2} \phi^{\prime}+f_{4} \frac{\partial}{\partial z}\left(\nabla^{2} H_{z}^{\prime}\right) \\
\frac{\partial H_{z}^{\prime}}{\partial t}=\frac{\partial w^{\prime}}{\partial z}+f_{10} \nabla^{2} H_{z}^{\prime}
\end{gathered}
$$

Here, the operator $\nabla$ represents the dimensionless gradient vector, whereas the dimensionless mathematical symbols $\nabla^{2}, \nabla_{2}^{2}$ and $\nabla^{4}$ denote the usual Laplacian, horizontal Laplacian and bi-Laplacian operators, respectively, where $\nabla=(\partial / \partial x, \partial / \partial y, \partial / \partial z), \nabla^{2}=\nabla_{2}^{2}+\partial^{2} / \partial z^{2}$, $\nabla_{2}^{2}=\partial^{2} / \partial x^{2}+\partial^{2} / \partial y^{2}$ and $\nabla^{4}=\nabla^{2}\left(\nabla^{2}\right)$.

\subsection{Normal Mode Analysis}

Due to the existence of periodic solutions, the normal mode analysis is adopted as a useful means to study the linear stability of the present nanofluidic system. Accordingly, the small quantities $w^{\prime}, T^{\prime}, \phi^{\prime}$ and $H_{z}^{\prime}$ can then be analyzed into two-dimensional waves by considering timedependent periodic disturbances, which are characterized by particular wave numbers $a_{x}$ and $a_{y}$.Therefore, the disturbances $w^{\prime}, T^{\prime}, \phi^{\prime}$ and $H_{z}^{\prime}$ can be written in the following form:

$$
\left[\begin{array}{l}
w^{\prime} \\
T^{\prime} \\
\phi^{\prime} \\
H_{z}^{\prime}
\end{array}\right]=\left[\begin{array}{c}
w(z) \\
\theta(z) \\
\Phi(z) \\
\mathcal{H}(z)
\end{array}\right] \exp \left[i\left(a_{x} x+a_{y} y\right)+\lambda t\right]
$$

Consequently, after substituting Eq. (54) into Eqs. (47), (48), (52) and (53), we get the following stability equations:

$$
\begin{gathered}
{\left[\begin{array}{c}
f_{1}\left(D^{4}-2 a^{2} D^{2}+a^{4}\right) w \\
-f_{2} R_{a} a^{2} \theta+f_{3} a^{2} \Phi
\end{array}\right]+f_{4}\left(D^{3}-a^{2} D\right) \mathcal{H}=\lambda\left(D^{2}-a^{2}\right) w} \\
w+\left(f_{5} D^{2}-f_{7} D-f_{5} a^{2}\right) \theta-f_{6} D \Phi=\lambda \theta \\
-\frac{N_{t}}{N_{b}} w+f_{9}\left(D^{2}-a^{2}\right) \theta+f_{8}\left(D^{2}-a^{2}\right) \Phi=\lambda \Phi \\
D w+f_{10}\left(D^{2}-a^{2}\right) \mathcal{H}=\lambda \mathcal{H}
\end{gathered}
$$

Here, $a$ shows the dimensionless wave number in the $x-y$ plane and $D^{n}$ represents the $n^{\text {th }}$ - order derivative with respect to the variable $z$, where $a=\left(a_{x}^{2}+a_{y}^{2}\right)^{0.5}$ and $D^{n}=d^{n} / d z^{n}$.

By assuming that the principle of exchange of stabilities is valid for the present thermo-magneto-hydrodynamic stability problem and the stationary convection (i.e., $\lambda=0$ ) is the solely considered mode, the system of Eqs. (55) - (58) can be reduce to the following eigenvalue problem:

$$
\left(\begin{array}{ccc}
A_{w} & 0 & f_{3} a^{2} \\
1 & B_{\theta} & B_{\Phi} \\
-N_{t} / N_{b} & C_{\theta} & C_{\Phi}
\end{array}\right)\left(\begin{array}{c}
w \\
\theta \\
\Phi
\end{array}\right)=R_{a}\left(\begin{array}{ccc}
0 & f_{2} a^{2} & 0 \\
0 & 0 & 0 \\
0 & 0 & 0
\end{array}\right)\left(\begin{array}{c}
w \\
\theta \\
\Phi
\end{array}\right)
$$

Additionally, the differential operators $A_{w}, B_{\theta}, B_{\Phi}, C_{\theta}$ and $C_{\Phi}$ shown above are expressed as follows:

$$
\begin{gathered}
A_{w}=f_{1} D^{4}-\left(2 f_{1} a^{2}+\frac{f_{4}}{f_{10}}\right) D^{2}+f_{1} a^{4} \\
B_{\theta}=f_{5} D^{2}-f_{7} D-f_{5} a^{2} \\
B_{\Phi}=-f_{6} D \\
C_{\theta}=f_{9}\left(D^{2}-a^{2}\right) \\
C_{\Phi}=f_{8}\left(D^{2}-a^{2}\right)
\end{gathered}
$$

In view of the linear stability theory and normal mode analysis, the boundary conditions (51) become:

$$
w=D w=\theta=D \Phi+\frac{N_{t}}{N_{b}} D \theta=0 \quad \text { at } \quad z=0,1
$$

From the computational point of view, the criterion for the onset magneto-convection in $\mathrm{Al}_{2} \mathrm{O}_{3}$ - water nanofluids can be examined efficiently after converting the Neumann boundary condition for the nanoparticles $D \Phi+\left(N_{t} / N_{b}\right) D \theta=0$ into a Dirichlet type boundary condition. For this purpose, it is more useful to apply a suitable change in the mathematical formulation of the problem by considering a new variable $\Gamma$ instead of $\Phi$. Based on the latest work of Wakif et al. (2018b), this modification can be easily made by setting the imposed change of variable $\Gamma=-D \Phi-\left(N_{t} / N_{b}\right) D \theta$. In view of this consideration, the eigenvalue problem described by Eq. (59) becomes:

$$
\begin{aligned}
\left(\begin{array}{ccc}
\bar{A}_{w} & -R_{N} N_{t} a^{2} / N_{b} & \bar{A}_{\Gamma} \\
f_{(\rho c)} & \bar{B}_{\theta} & N_{b} \\
\bar{C}_{w} & 0 & \bar{C}_{\Gamma}
\end{array}\right)\left(\begin{array}{c}
w \\
\theta \\
\Gamma
\end{array}\right)= \\
R_{a}\left(\begin{array}{ccc}
0 & f_{(\rho \beta)} a^{2} & 0 \\
0 & 0 & 0 \\
0 & 0 & 0
\end{array}\right)\left(\begin{array}{c}
w \\
\theta \\
\Gamma
\end{array}\right)
\end{aligned}
$$

Here, the modified differential operators $\bar{A}_{w}, \bar{A}_{\Gamma}, \bar{B}_{\theta}, \bar{C}_{w}$ and $\bar{C}_{\Gamma}$ are given explicitly by :

$$
\begin{gathered}
\bar{A}_{w}=f_{\mu} D^{4}-\left(2 f_{\mu} a^{2}+Q f_{\sigma} f_{\bar{\mu}}^{2}\right) D^{2}+\left(f_{\mu} a^{4}-\frac{R_{N} N_{t} L_{e}}{N_{b}}\right) \\
\bar{A}_{\Gamma}=-R_{N} D \\
\bar{B}_{\theta}=f_{k}\left(D^{2}-a^{2}\right)
\end{gathered}
$$




$$
\begin{gathered}
\bar{C}_{w}=\frac{L_{e} N_{t}}{N_{b}} D \\
\bar{C}_{\Gamma}=D^{2}-a^{2}
\end{gathered}
$$

For the resulting eigenvalue problem (66), the associated boundary conditions are written as follows:

$$
w=D w=\theta=\Gamma=0 \quad \text { at } \quad z=0,1
$$

Table 2 Computed values of $f_{\rho}, f_{\mu}, f_{\bar{\mu}}, f_{k}, f_{\sigma}, f_{(\rho \beta)}$ and $f_{(\rho c)}$, for different values of $d_{n p}$ and $\phi_{0}$.

\begin{tabular}{ccccccccc}
\hline$d_{n p}$ & $\phi_{0}$ & $f_{\rho}$ & $f_{\mu}$ & $f_{\bar{\mu}}$ & $f_{k}$ & $f_{\sigma}$ & $f_{(\rho \beta)}$ & $f_{(\rho c)}$ \\
\hline \multirow{3}{*}{$30 \mathrm{~nm}$} & 0.01 & 1.02981 & 1.08960 & 0.99999 & 1.04922 & 0.98507 & 0.99161 & 0.99728 \\
& 0.02 & 1.05963 & 1.20181 & 0.99999 & 1.08255 & 0.97029 & 0.98322 & 0.99457 \\
& 0.03 & 1.08944 & 1.34222 & 0.99999 & 1.11171 & 0.95566 & 0.97483 & 0.99186 \\
& 0.04 & 1.11926 & 1.52185 & 0.99999 & 1.13845 & 0.94117 & 0.96644 & 0.98915 \\
\hline \multirow{3}{*}{$35 \mathrm{~nm}$} & 0.01 & 1.02981 & 1.08520 & 0.99999 & 1.04650 & 0.98507 & 0.99161 & 0.99728 \\
& 0.02 & 1.05963 & 1.19095 & 0.99999 & 1.07798 & 0.97029 & 0.98322 & 0.99457 \\
& 0.03 & 1.08944 & 1.32178 & 0.99999 & 1.10553 & 0.95566 & 0.97483 & 0.99186 \\
& 0.04 & 1.11926 & 1.48679 & 0.99999 & 1.13079 & 0.94117 & 0.96644 & 0.98915 \\
\hline \multirow{3}{*}{$40 \mathrm{~nm}$} & 0.01 & 1.02981 & 1.08158 & 0.99999 & 1.04426 & 0.98507 & 0.99161 & 0.99728 \\
& 0.02 & 1.05963 & 1.18208 & 0.99999 & 1.07423 & 0.97029 & 0.98322 & 0.99457 \\
& 0.03 & 1.08944 & 1.30529 & 0.99999 & 1.10046 & 0.95566 & 0.97483 & 0.99186 \\
& 0.04 & 1.11926 & 1.45890 & 0.99999 & 1.12450 & 0.94117 & 0.96644 & 0.98915 \\
\hline \multirow{3}{*}{$45 \mathrm{~nm}$} & 0.01 & 1.02981 & 1.07853 & 0.99999 & 1.04238 & 0.98507 & 0.99161 & 0.99728 \\
& 0.02 & 1.05963 & 1.17466 & 0.99999 & 1.07108 & 0.97029 & 0.98322 & 0.99457 \\
& 0.03 & 1.08944 & 1.29160 & 0.99999 & 1.09618 & 0.95566 & 0.97483 & 0.99186 \\
& 0.04 & 1.11926 & 1.43602 & 0.99999 & 1.11921 & 0.94117 & 0.96644 & 0.98915 \\
\hline
\end{tabular}

Table 3 Effective values of the parameters $R_{N}, L_{e}, N_{b}$ and $N_{t}$, for different values of $d_{n p}$ and $\phi_{0}$.

\begin{tabular}{cccccc}
\hline$d_{n p}$ & $\phi_{0}$ & $R_{N}$ & $L_{e}\left(\times 10^{4}\right)$ & $N_{b}\left(\times 10^{-6}\right)$ & $N_{t}\left(\times 10^{-6}\right)$ \\
\hline \multirow{3}{*}{$30 \mathrm{~nm}$} & 0.01 & 0.15336 & 0.89376 & 0.81548 & 0.56988 \\
& 0.02 & 0.30672 & 0.89376 & 1.63097 & 1.13976 \\
& 0.03 & 0.46008 & 0.89376 & 2.44646 & 1.70964 \\
& 0.04 & 0.61344 & 0.89376 & 3.26194 & 2.27952 \\
\hline \multirow{3}{*}{$35 \mathrm{~nm}$} & 0.01 & 0.15336 & 1.04272 & 0.69898 & 0.56988 \\
& 0.02 & 0.30672 & 1.04272 & 1.39797 & 1.13976 \\
& 0.03 & 0.46008 & 1.04272 & 2.09696 & 1.70964 \\
$40 \mathrm{~nm}$ & 0.04 & 0.61344 & 1.04272 & 2.79595 & 2.27952 \\
\hline & 0.01 & 0.15336 & 1.19168 & 0.61161 & 0.56988 \\
& 0.02 & 0.30672 & 1.19168 & 1.22323 & 1.13976 \\
& 0.03 & 0.46008 & 1.19168 & 1.83484 & 1.70964 \\
& 0.04 & 0.61344 & 1.19168 & 2.44646 & 2.27952 \\
\hline \multirow{3}{*}{$\mathrm{nm}$} & 0.01 & 0.15336 & 1.34064 & 0.54365 & 0.56988 \\
& 0.02 & 0.30672 & 1.34064 & 1.08731 & 1.13976 \\
& 0.03 & 0.46008 & 1.34064 & 1.63097 & 1.70964 \\
& 0.04 & 0.61344 & 1.34064 & 2.17463 & 2.27952 \\
\hline
\end{tabular}




\section{NUMERICAL SOLUTION}

\subsection{Solution Methodology}

As already mentioned, the obtained linear eigenvalue problem (66) in association with the reduced boundary conditions (72) can be handled numerically by discretizing their ordinary differential equations along the $z$-direction. Consequently, after choosing an appropriate discretization scheme, the continuous stationary differential equations arising from the present stability problem are treated mathematically with their associated boundary conditions (72) as a generalized algebraic eigenvalue problem, whose eigenvalues constitute a discrete set of all possible values of the control parameter $R_{a}$. In this regards, it bears mentioning here that the thermal Rayleigh number $R_{a}$ corresponding to a fixed wave number $a$ is selected in such a way to be the smallest value among all positive real eigenvalues. Moreover, this possible value depends greatly on the values of the control parameters $\phi_{0}, d_{n p}$ and $Q$ characterizing the nanofluidic system.

Accordingly, in order to find the underlying relationship between the thermal Rayleigh number $R_{a}$ and the wave number $a$ corresponding to the stationary mode of convection, we adopt an efficient tool that can yield more accurate results. In view of this, the physical domain is converted from $\left[\begin{array}{ll}0 & 1\end{array}\right]$ to the space interval $\left[\begin{array}{ll}-1 & 1\end{array}\right]$ by replacing the space variable $z$ with another variable $\xi$, where $\xi=2 z-1$. At this stage, the main unknowns $w, \theta$ and $\Gamma$ are transformed to $\bar{w}, \bar{\theta}$ and $\bar{\Gamma}$, respectively, such that $\bar{w}=w(z(\xi)), \bar{\theta}=\theta(z(\xi)), \bar{\Gamma}=\Gamma(z(\xi))$ and $D^{n} X=2^{n} \bar{D}^{n} \bar{X}$, where $n$ is an integer derivative order and $X$ is an unknown of the problem (i. e., $w, \theta$ or $\Gamma$ ) with $\bar{D}^{n}=d^{n} / d \xi^{n}$. Keeping in mind this mathematical considerations, the computational domain [-1 1] is discretized non-uniformly into a set of $(N-1)$ successive sub-domains $\left[\xi_{i+1} \xi_{i}\right]$ by utilizing the Gauss-Lobatto grid collocation points, in such a way that $\xi_{i}=\cos [\pi(i-1) /(N-1)]$ and $\xi_{N} \leq \xi_{i} \leq \xi_{1}$, where $i$ is an integer index varying decreasingly from $(N-1)$ to 1 and $N$ is the total number of collocation points chosen in the numerical implementation.

Hence, based on the Gauss-Lobatto grid collocation points and the Chebyshev polynomial interpolation, the derivatives of the function $\bar{X}(\xi)$ with respect to the variable $\xi$ at a collocation point $\xi_{i}$ are expressed by:

$$
\bar{D}^{n} \bar{X}\left(\xi_{i}\right)=\sum_{j=1}^{N} \tilde{d}_{i j}^{(n)} \bar{X}\left(\xi_{j}\right)=\sum_{j=1}^{N} \tilde{d}_{i j}^{(n)} \bar{X}_{j}
$$

In addition, the discrete solutions $\left\{\left(\bar{w}_{i}, \bar{\theta}_{i}, \bar{\Gamma}_{i}\right) / 2 \leq i \leq N-1\right\}$ at each collocation point $\xi_{i}$ can be found through the following resulting algebraic eigenvalue problem:

$$
\begin{array}{rcc}
\left(\begin{array}{ccc}
\bar{A}_{i j}^{\bar{w}} & -R_{N} N_{t} a^{2} \delta_{i j} / N_{b} & \bar{A}_{i j}^{\bar{\Gamma}_{j}} \\
f_{(\rho c)} \delta_{i j} & \bar{B}_{i j}^{\bar{\theta}} & N_{b} \delta_{i j} \\
\bar{C}_{i j}^{\bar{w}} & Z_{i j} & \bar{C}_{i j} \bar{\Gamma}^{\bar{v}}
\end{array}\right)\left(\begin{array}{c}
\bar{w}_{j} \\
\bar{\theta}_{j} \\
\bar{\Gamma}_{j}
\end{array}\right)= \\
\\
R_{a}\left(\begin{array}{ccc}
Z_{i j} & f_{(\rho \beta)} a^{2} \delta_{i j} & Z_{i j} \\
Z_{i j} & Z_{i j} & Z_{i j} \\
Z_{i j} & Z_{i j} & Z_{i j}
\end{array}\right)\left(\begin{array}{c}
\bar{w}_{j} \\
\bar{\theta}_{j} \\
\bar{\Gamma}_{j}
\end{array}\right)
\end{array}
$$

Here, $\delta_{i j}$ represents the Kronecker symbol, where $2 \leq i, j \leq N-1$. Furthermore, the elements $\bar{A}_{i j}^{\bar{w}}, \bar{A}_{i j}^{\bar{\Gamma}}, \bar{B}_{i j}^{\bar{\theta}}, \bar{C}_{i j}^{\bar{w}}, \bar{C}_{i j}^{\bar{\Gamma}}, Z_{i j}$ shown above are expressed formally by:

$$
\begin{aligned}
\bar{A}_{i j}^{\bar{w}}=16 f_{\mu} \widetilde{D}_{i j}^{(4)}+\left[\begin{array}{l}
-4\left(2 f_{\mu} a^{2}+Q f_{\sigma} f_{\bar{\mu}}^{2}\right) \widetilde{D}_{i j}^{(2)} \\
+\left(f_{\mu} a^{4}-\frac{R_{N} N_{t} L_{e}}{N_{b}}\right) \delta_{i j}
\end{array}\right] \\
\bar{A}_{i j}^{\bar{\Gamma}}=-2 R_{N} \tilde{d}_{i j}^{(1)}
\end{aligned}
$$

$$
\begin{gathered}
\bar{B}_{i j}^{\bar{\theta}}=f_{\kappa}\left(4 \tilde{d}_{i j}^{(2)}-a^{2} \delta_{i j}\right) \\
\bar{C}_{i j}^{\bar{\omega}}=\frac{2 L_{e} N_{t}}{N_{b}} \widetilde{D}_{i j}^{(1)} \\
\bar{C}_{i j}^{\bar{\Gamma}}=4 \tilde{d}_{i j}^{(2)}-a^{2} \delta_{i j} \\
Z_{i j}=0
\end{gathered}
$$

According to Canuto et al. (2012), the elements $\tilde{d}_{i j}^{(1)}$ of the firstorder Chebyshev differentiation matrix $\widetilde{\mathbf{D}}^{(\mathbf{1})}$ are given by:

$$
\tilde{d}_{i j}^{(1)}= \begin{cases}\left(2 N^{2}-4 N+3\right) / 6 & \text { for } i=j=1 \\ \xi_{i} /\left(2 \xi_{i}^{2}-2\right) & \text { for } i=j \neq 1, N \\ (-1)^{i+j} c_{i} /\left(c_{j} \xi_{i}-c_{j} \xi_{j}\right) & \text { for } i \neq j \\ \left(-2 N^{2}+4 N-3\right) / 6 & \text { for } i=j=N\end{cases}
$$

Here, the coefficients $c_{i}$ are defined as follows:

$$
c_{i}= \begin{cases}2 & \text { for } i=1, N \\ 1 & \text { for } i \neq 1, N\end{cases}
$$

In view of the Chebyshev-Gauss-Lobatto Spectral Method, the other elements $\tilde{d}_{i j}^{(n)}$ corresponding to the $n^{t h}$-order Chebyshev differentiation matrix $\widetilde{\mathbf{D}}^{(\mathbf{n})}$ can be computed recurrently as follows:

$$
\tilde{d}_{i j}^{(n)}=\sum_{k=1}^{N} \tilde{d}_{i k}^{(n-1)} \tilde{d}_{k j}^{(1)}
$$

Based on the mathematical techniques reported by Trefethen (2000) and the boundary conditions $w=0$ and $D w=0$, the other elements $\widetilde{D}_{i j}^{(1)}, \widetilde{D}_{i j}^{(2)}$ and $\widetilde{D}_{i j}^{(4)}$ used above are computed analytically from the basic elements $\tilde{d}_{i j}^{(1)}, \tilde{d}_{i j}^{(2)}, \tilde{d}_{i j}^{(3)}$ and $\tilde{d}_{i j}^{(4)}$ as follows :

$$
\begin{gathered}
\widetilde{D}_{i j}^{(1)}=\frac{1}{\left(1-\xi_{i}^{2}\right)}\left[\left(1-\xi_{i}^{2}\right) \tilde{d}_{i j}^{(1)}-2 \xi_{i} \delta_{i j}\right] \\
\widetilde{D}_{i j}^{(2)}=\frac{1}{\left(1-\xi_{i}^{2}\right)}\left[\left(1-\xi_{i}^{2}\right) \tilde{d}_{i j}^{(2)}-4 \xi_{i} \tilde{d}_{i j}^{(1)}-2 \delta_{i j}\right] \\
\widetilde{D}_{i j}^{(4)}=\frac{1}{\left(1-\xi_{i}^{2}\right)}\left[\left(1-\xi_{i}^{2}\right) \tilde{d}_{i j}^{(4)}-8 \xi_{i} \tilde{d}_{i j}^{(3)}-12 \tilde{d}_{i j}^{(2)}\right]
\end{gathered}
$$

As proved above, the generalized algebraic eigenvalue problem described by Eq. (74) has $(3 \mathrm{~N}-6)$ eigenvalues $\gamma_{i}$. These characteristic values depend implicitly upon several parameters, including the wave number $a$, the volumetric fraction of nanoparticles $\phi_{0}$, the diameter of nanoparticles $d_{n p}$ as well as the magnetic Chandrasekhar number $Q$. Hence, for specified values of these physical parameters, the possible eigenvalues $\gamma_{i}$ can be found numerically for each fixed wave number $a$, in such a way that the sought thermal Rayleigh number $R_{a}$ must be the smallest eigenvalue among the whole set of positive real eigenvalues (i.e., $\left.R_{a}=\min \left\{\gamma_{i} / \gamma_{i}>0\right\}\right)$. After employing the Golden Section Search Method (GSSM), we can find the critical stability parameters $R_{a c}$ and $a_{c}$ by minimizing numerically $R_{a}$ with respect to $a$ in a wide interval $\left[a_{i} a_{f}\right]$ (e.g., $\left.a \in\left[\begin{array}{ll}0 & 10\end{array}\right]\right)$.

\subsection{Numerical, Analytical and Semi-Analytical Validations}

As discussed above, for analyzing the thermo-magneto-hydrodynamic instability occurred in a thin horizontal layer of a nanofluid, several numerical implementations have been performed for of $\mathrm{Al}_{2} \mathrm{O}_{3}$ - water nanofluids using Chebyshev-Gauss-Lobatto Spectral Method (CGLSM). Recently, this method attracts extensive attention from researchers due to its importance in a wide range of applications as a fast, flexible and accurate numerical procedure to solve the linear and nonlinear problems 
in fluid mechanics and other related areas. Hence, in order to verify the correctness and reliability of our present numerical findings in terms of the critical stability parameters $R_{a c}$ and $a_{c}$, a detailed side-by-side comparison has been carried out using Chebyshev-Gauss-Lobatto Spectral Method (CGLSM) and Generalized Differential Quadrature Method (GDQM). Moreover, it is worth mentioning here that GDQM is exceptionally used in this investigation as a comparative numerical method to validate our results. To the best of our knowledge, no previous studies have systematically examined by means of GDQM to solve similar stability problems. Hence, in order to provide extensive details about this numerical method, the readers can refer to the book of Shu (2012). Also, the interested researchers can see the innovative works of Fidanoglu et al. (2014), Qasim et al. (2018) and the references therein, in which GDQM is explained more fully through two different practical situations. Based on our CGLSM and GDQM codes, the results are presented in tabular and graphical forms to discuss the significant effects of the emerging parameters $Q, \phi_{0}$ and $d_{n p}$ on the thermo-magnetohydrodynamic stability of $\mathrm{Al}_{2} \mathrm{O}_{3}$ - water nanofluids as well as to quantify the agreement between the results of these numerical methods. As shown in Fig. 2 , Fig. 3 , Fig. 4 , Fig. 5 , Fig. 6 and Table 4, it is found that the graphical and tabular results given by CGLSM are very close to those of GDQM, which lends further credibility to our final outcomes and also emphasizes the robustness and validity of the CGLSM code.

In the aim to authenticate the accuracy of our numerical results generated by the aforementioned collocation numerical methods and verify the efficiency of the computational codes used in this investigation towards some limiting cases for computing the critical values of the parameters $R_{a}$ and a, we first compare the results of our CGLSM and GDQM codes with those obtained analytically by Chandrasekhar (1961) and numerically by Wakif et al. (2018b) using Variational Method (VM) and Runge-Kutta-Fehlberg Method (RKFM), respectively, for the case of electrically conducting fluids (i. e. , $\phi_{0}=0$ ) as shown in Table 5.
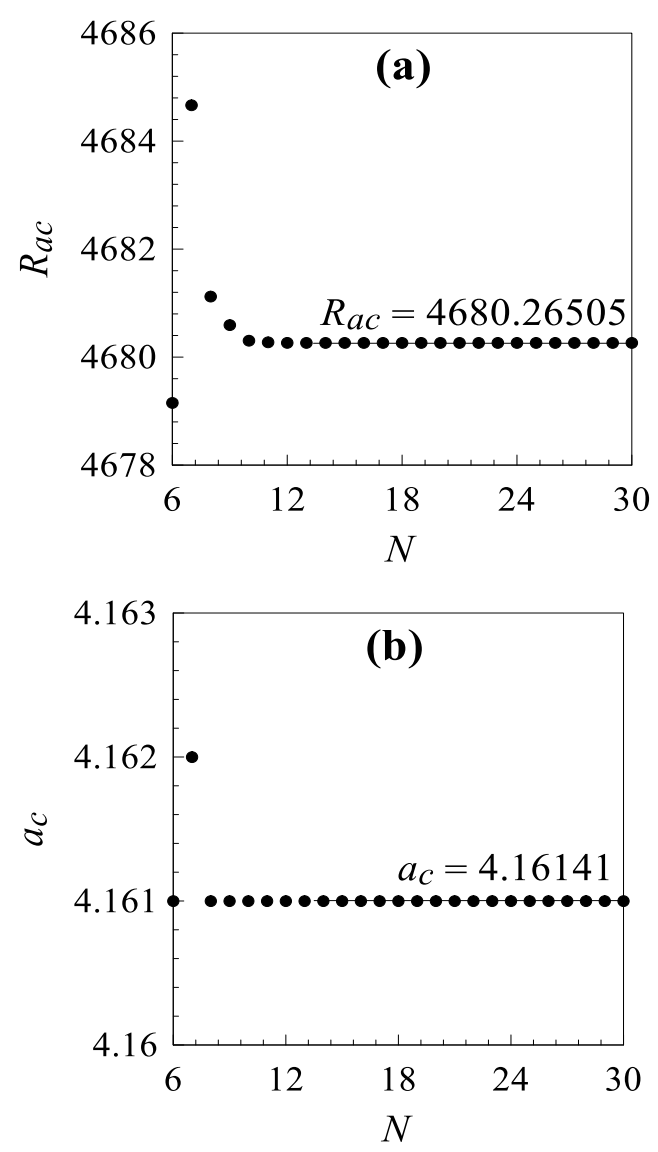
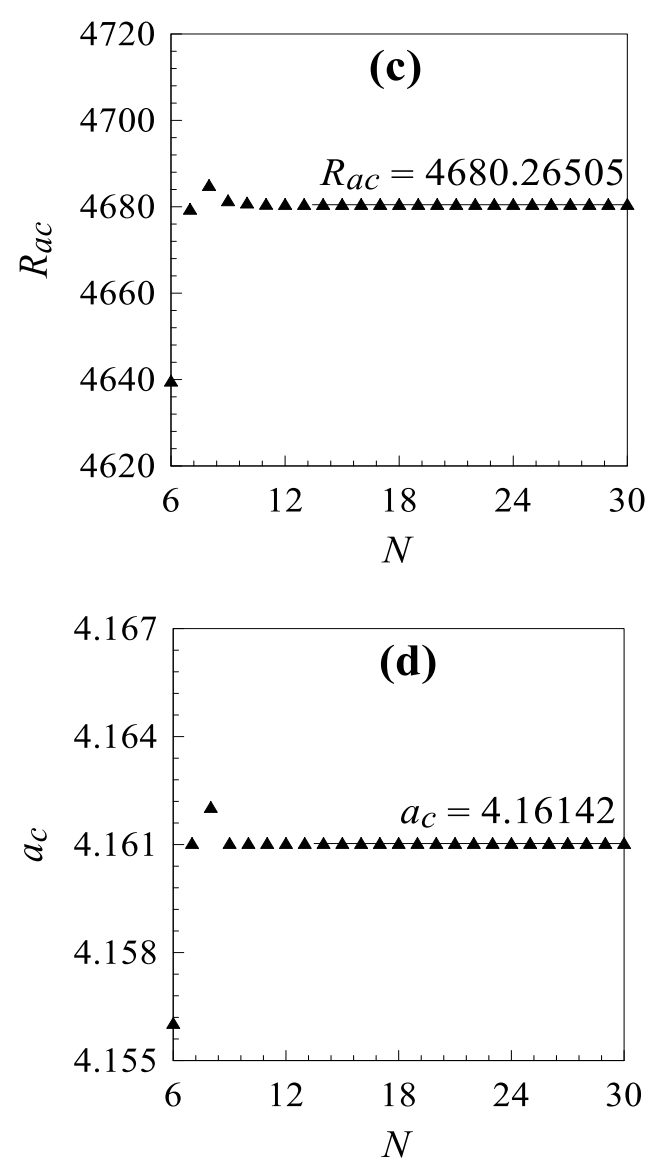

Fig. 2 Convergence test for $R_{a c}$ and $a_{c}$ using (a,b) CGLSM and (c,d) GDQM, when $Q=200, \phi_{0}=0.01$ and $d_{n p}=30 \mathrm{~nm}$.

In the second set of examination tests, we compare in Table 6 our numerical results given by CGLSM and GDQM with those obtained semi-analytically with the help of Wakif-Galerkin Weighted Residuals Technique (WGWRT) based on the revised Buongiorno's two-phase classical model, in the case of electrically conducting nanofluids, where $f_{\rho}=f_{\mu}=f_{\bar{\mu}}=f_{k}=f_{\sigma}=f_{(\rho \beta)}=f_{(\rho c)}=1$.

For a more explanation and detailed description of WGWRT, the interested reader is strongly encouraged to refer to the pioneering work of Wakif et al. (2018c), in which WGWRT is used as a semianalytical method for analyzing the onset of electro-convection in dielectric nanofluids.

As expected, it is clearly seen from Table 5 and Table 6 that the numerical results of CGLSM and GDQM have an excellent degree of agreement with those of VM, RKFM and WGWRT. Consequently, the validation of our results is confirmed numerically, analytically and semianalytically with a very high degree of precision.

Moreover, Fig. 2 is plotted for $\mathrm{Al}_{2} \mathrm{O}_{3}$ - water nanofluids to control the convergence efficiency of CGLSM and GDQM in terms of the critical stability parameters $R_{a c}$ and $a_{c}$. From this graphical representation, it is clearly observed that the convergence curves obtained by CGLSM and GDQM can be stabilized when the number of collocation points $N$ exceeds a certain value $N_{0}$. As a result, it is noticed graphically from Fig. 2 that $N_{0}=10$, in the case where $Q=200, \phi_{0}=0.01$ and $d_{n p}=30 \mathrm{~nm}$.

In order to achieve an absolute accuracy of the order of $10^{-6}$, it is more recommended to take $N=28$ for the number of collocation points during all subsequent analyses by CGLSM and GDQM. Furthermore, it 
is found that the average CPU time taken for computing the accurate critical stability parameters $R_{a c}$ and $a_{c}$ by CGLSM or GDQM is no more than $40 \mathrm{~s}$.

\section{ANALYSIS OF RESULTS}

In the present numerical investigation, the onset of thermo-magnetohydrodynamic instability in $\mathrm{Al}_{2} \mathrm{O}_{3}$ - water nanofluids is analyzed more realistically for non-slip, zero nanoparticles mass flux and isothermal boundary conditions, in such a way that the effects of Brownian motion and thermophoresis of nanoparticles are taken into account by using Buongiorno's two-phase nanofluid model with variable thermophysical properties.

Based on the linear stability analysis and the normal mode method, the set of the governing partial differential equations along with their corresponding boundary conditions are carefully transformed into a generalized algebraic eigenvalue problem with the help of ChebyshevGauss-Lobatto Spectral Method (CGLSM), in order to solve this problem numerically by means of the Golden Section Search Method (GSSM). Additionally, all the results given by our CGLSM algorithm are validated numerically against those obtained using Generalized Differential Quadrature Method (GDQM).

The generated CGLSM and GDQM codes used in this investigation are further tested and multiply validated (i.e., numerically, analytically and semi-analytically) for some limiting cases using other powerful existing methods, such as Variational Method (VM), Runge-KuttaFehlberg Method (RKFM) and Wakif-Galerkin Weighted Residuals Technique (WGWRT).

Moreover, the effects of some parameters including the magnetic Chandrasekhar number $Q$, the volumetric fraction $\phi_{0}$ and the diameter $d_{n p}$ of alumina nanoparticles on the onset of magneto-convection in a thin horizontal layer filled with alumina - water nanofluid are examined numerically by CGLSM and GDQM via various graphical illustrations as highlighted in Fig. 3 and Fig. 4. In these figures, the variations of the critical stability parameters $R_{a c}$ and $a_{c}$ as a function of the magnetic Chandrasekhar number $Q$ are plotted and discussed for multiple values of the pertinent parameters $\phi_{0}$ and $d_{n p}$, where $200 \leq Q \leq 1000$, $0.01 \leq \phi_{0} \leq 0.04$ and $30 \mathrm{~nm} \leq d_{n p} \leq 45 \mathrm{~nm}$.

Furthermore, it is worth mentioning here that the graphical curves and tabular results highlighted in Fig. 3 , Fig. 4 and Table 4 are reillustrated in another way in Fig. 5 and Fig. 6, in order to facilitate the stability analysis of $\mathrm{Al}_{2} \mathrm{O}_{3}$ - water nanofluids.

Additionally, the rates of increase or decrease for the tabulated functions $R_{a c}=g_{1}(Q), a_{c}=h_{1}(Q), R_{a c}=g_{2}\left(\phi_{0}\right), a_{c}=h_{2}\left(\phi_{0}\right)$, $R_{a c}=g_{3}\left(d_{n p}\right), a_{c}=h_{3}\left(d_{n p}\right)$ and $R_{a c}=f\left(a_{c}\right)$ are estimated numerically by computing the slopes of their corresponding data, respectively, using the linear regression model (Makinde et al., 2018 ; Animasaun and Pop , 2017; Shah et al., 2018) as shown in Table 4.

The effect of the presence of an externally applied magnetic field on the critical stability parameters $R_{a c}$ and $a_{c}$ characterizing the onset of magneto-convection in $\mathrm{Al}_{2} \mathrm{O}_{3}$ - water nanofluids is exhibited clearly in Fig. 3 and Fig. 4 by varying the magnetic Chandrasekhar number $Q$. In these graphical illustrations, the variations of $R_{a c}$ and $a_{c}$ are depicted as a function of the magnetic Chandrasekhar number $Q$, for different values of the parameters $\phi_{0}$ and $d_{n p}$.

As seen in Fig. 3 and Fig. 4, it is obviously observed that the critical thermal Rayleigh number $R_{a c}$ increases with the simultaneous elevation of the magnetic Chandrasekhar number $Q$. Physically, this fact is mainly happened due to the Lorentzian magnetic drag force generated by the magnetic field in the electrically conducting medium. Likewise, this magnetic force has a resistive behavior, which acts to oppose and slow down the nanofluid flow caused by the imposed heating gradient $\Delta T / L$.
In view of this, it is concluded that the thermo-magneto-hydrodynamic stability of $\mathrm{Al}_{2} \mathrm{O}_{3}$-water nanofluid can be affected by applying a uniform transverse magnetic field to a horizontal thin layer of this electrically conducting nanofluid. Hence, the onset of magneto-convection in the medium can be delayed under the influence of the magnetic field strength $H_{0}$, in such a way that the nanofluidic system becomes more stable with increasing the magnetic Chandrasekhar number $Q$. Similarly, it is noticed from the same figures that the critical wave number $a_{c}$ can be increased by intensifying the magnetic field strength $H_{0}$, indicating that the thermomagneto-hydrodynamic stability of $\mathrm{Al}_{2} \mathrm{O}_{3}$ - water nanofluids caused by the increase in the magnetic Chandrasekhar number $Q$ is accompanied by a significant reduction in the critical size of convection cells $L_{c}$ (i.e., $L_{c}=2 \pi / a_{c}$ ).

As shown in Table 2 and Table 3, the effect of adding alumina nanoparticles $\mathrm{Al}_{2} \mathrm{O}_{3}$ into a pure water $\mathrm{H}_{2} \mathrm{O}$ has a major influence on the thermophysical properties of the resulting nanofluid. This subsequently affects the physical control parameters $R_{N}, N_{b}$ and $N_{t}$ characterizing respectively the significant impact of nanoparticles volume fraction, Brownian motion and thermophoresis of alumina nanoparticles on the thermo-magneto-hydrodynamic stability of $\mathrm{Al}_{2} \mathrm{O}_{3}$ - water nanofluids.

From Table 2, it can be concluded that the increasing amount of alumina nanoparticles $\mathrm{Al}_{2} \mathrm{O}_{3}$ inserted into the pure water is the most important factor responsible for significant enhancement in the heat transfer rate efficiency of $\mathrm{Al}_{2} \mathrm{O}_{3}$ - water nanofluid, due to the fact that the thermal conductivity of this nanofluid is an increasing function of the volumetric fraction $\phi_{0}$. In view of this fact, it can be concluded from Table 3 that raising the volumetric fraction $\phi_{0}$ of alumina nanoparticles $\mathrm{Al}_{2} \mathrm{O}_{3}$ brings about a noteworthy boost in the Brownian motion and thermophoresis effects, which yields significant random movement of alumina nanoparticles $\mathrm{Al}_{2} \mathrm{O}_{3}$ throughout the whole medium. Therefore, keeping that in mind, the nanofluidic system becomes more unstable due to the intensive disturbances exerted by the nanoparticles flow in the nanofluid layer. Hence, the volumetric fraction of nanoparticles $\phi_{0}$ has a destabilizing effect on the nanofluidic system. This physical analysis is consistent with the graphical results depicted in Fig. 3, in which the onset of magneto-convection in alumina-water nanofluids can be hasten by adding alumina nanoparticles $\mathrm{Al}_{2} \mathrm{O}_{3}$ in the nanofluidic medium in very small amounts. Also, it is revealed from this graphical content that there is a marked enhancement in the critical size of convection cells $L_{c}$. These results are happened due to the significant decrease in the critical stability parameters $R_{a c}$ and $a_{c}$ during the concentration increase.

Fig. 4 is plotted to explore the influence of alumina nanoparticles dimension $d_{n p}$ on the critical values $R_{a c}$ and $a_{c}$ characterizing the onset of thermo-magneto-hydrodynamic instability and the critical size of convection cells, respectively. As for the previous graphical illustrations, it is observed similar behaviors in Fig. 4 for the diameter $d_{n p}$ of alumina nanoparticles $\mathrm{Al}_{2} \mathrm{O}_{3}$ on the critical stability parameters $R_{a c}$ and $a_{c}$. Consequently, the rise in the diameter $d_{n p}$ has a destabilizing effect on the nanofluidic system. Besides this, the onset of the magneto-convection in alumina - water nanofluids is accelerated and the critical size of convection cells $L_{c}$ is increased due to the increase in the diameter $d_{n p}$. Additionally, it can be noticed from the expressions of Lewis number $L_{e}$ and Table 3 that there is a direct proportionality between $L_{e}$ and $d_{n p}$. Therefore, these parameters have the same influence on the thermomagneto-hydrodynamic stability of $\mathrm{Al}_{2} \mathrm{O}_{3}$ - water nanofluids.

As a remark, it is worth mentioning here that the limiting case of electrically conducting fluids is highlighted graphically by $\phi_{0}=0$ and $d_{n p}=0$ as shown in Fig. 3 and Fig. 4 , respectively. Based on these graphical presentations, it can be noted that the electrically conducting fluids are more stable than the electrically conducting nanofluids. Also, the key results arising from the present numerical investigation are summarized graphically as shown in Fig. 5 and Fig. 6. 

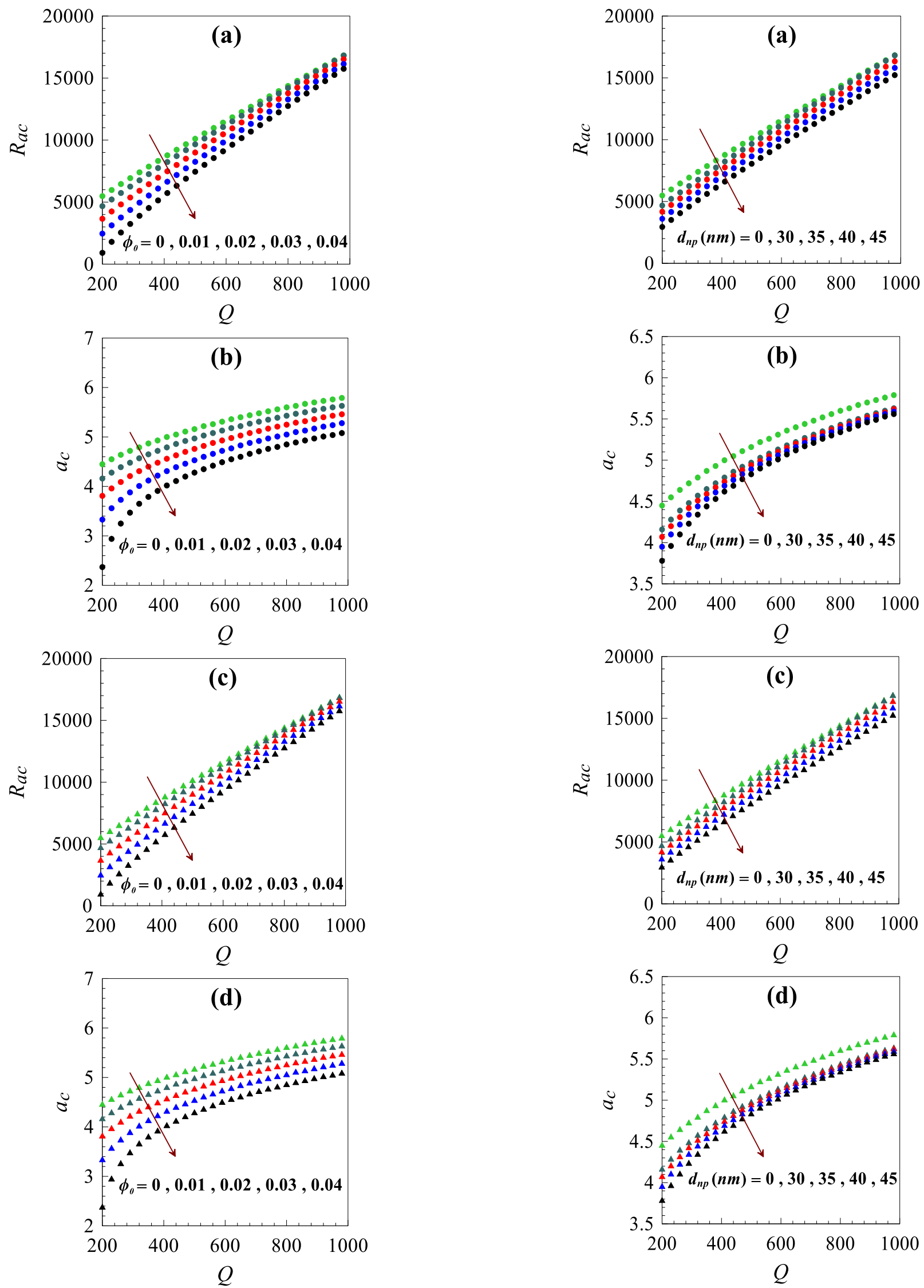

Fig. 3 Examination of the influence of $Q$ and $\phi_{0}$ on $R_{a c}$ and $a_{c}$ by means of (a,b) CGLSM and (c,d) GDQM, when $d_{n p}=30 \mathrm{~nm}$.

Fig. 4 Examination of the influence of $Q$ and $d_{n p}$ on $R_{a c}$ and $a_{c}$ by means of (a,b) CGLSM and (c,d) GDQM, when $\phi_{0}=0.01$. 

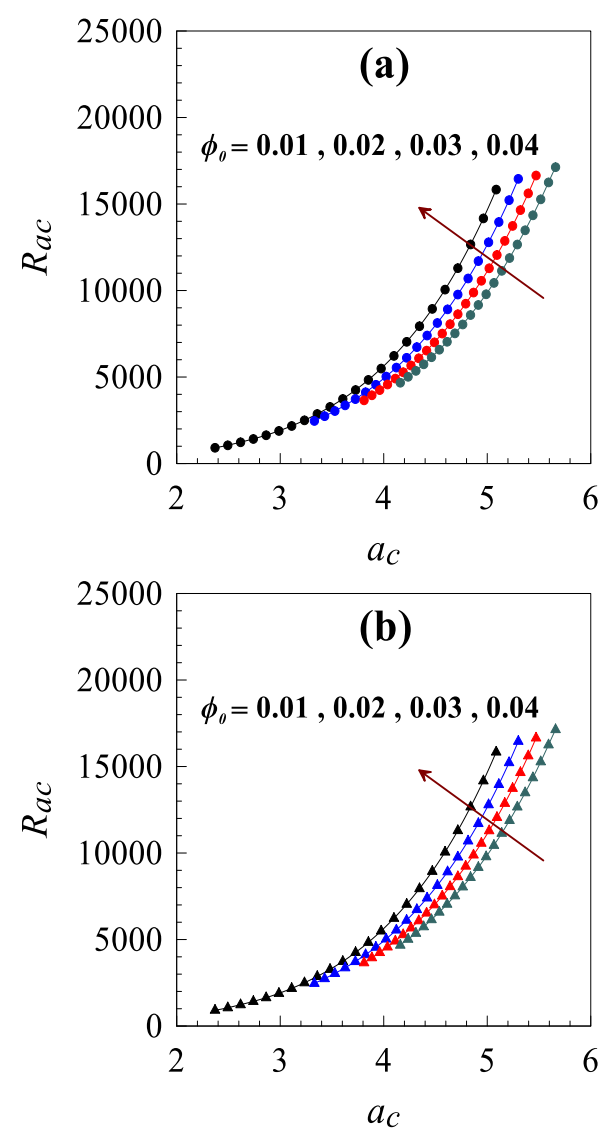

Fig. 5 Numerical determination of the variation of $R_{a c}$ with respect to $a_{c}$, for increasing values of $Q(200 \leq Q \leq 1000)$ and various values of $\phi_{0}$ using (a) CGLSM and (b) GDQM, when $d_{n p}=30 \mathrm{~nm}$.
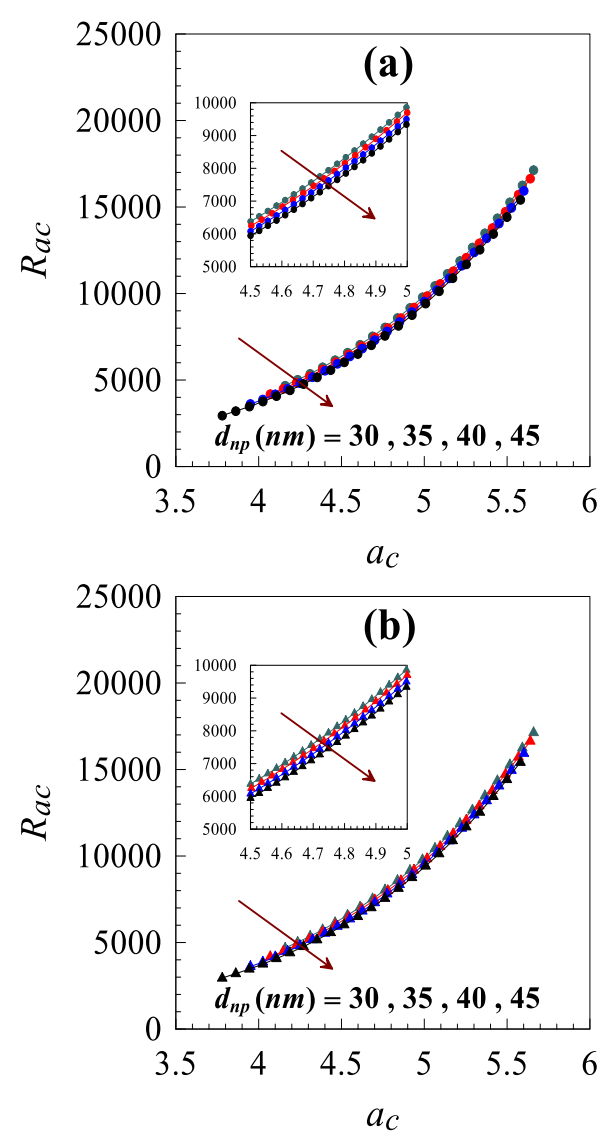

Fig. 6 Numerical determination of the variation of $R_{a c}$ with respect to $a_{c}$, for increasing values of $Q(200 \leq Q \leq 1000)$ and various values of $d_{n p}$ using (a) CGLSM and (b) GDQM, when $\phi_{0}=0.01$.

Table 4 Numerical estimation of $R_{a c}$ and $a_{c}$ with the help of CGLSM and GDQM, for various values of $Q, \phi_{0}$ and $d_{n p}$.

\begin{tabular}{|c|c|c|c|c|c|c|}
\hline \multirow{3}{*}{$Q$} & \multirow{3}{*}{$\phi_{0}$} & \multirow{3}{*}{$d_{n p}(n m)$} & \multicolumn{4}{|c|}{ Present Numerical Results } \\
\hline & & & \multicolumn{2}{|c|}{ CGLSM } & \multicolumn{2}{|c|}{ GDQM } \\
\hline & & & $R_{a c}$ & $a_{c}$ & $R_{a c}$ & $a_{c}$ \\
\hline 200 & \multirow{4}{*}{0.01} & \multirow{4}{*}{30} & 4680.26505 & 4.16141 & 4680.26505 & 4.16142 \\
\hline 500 & & & 9665.04216 & 4.97386 & 9665.04216 & 4.97383 \\
\hline 700 & & & 12720.09144 & 5.29646 & 12720.09144 & 5.29649 \\
\hline 1000 & & & 17107.11916 & 5.65539 & 17107.11916 & 5.65540 \\
\hline \multicolumn{3}{|c|}{ Slope1 for $R_{a c}=g_{1}(Q)$ and $a_{c}=h_{1}(Q)$} & 15,51837 & 0,00185 & 15,51837 & 0,00185 \\
\hline \multicolumn{3}{|c|}{ Slope2 for $R_{a c}=f\left(a_{c}\right)$} & \multicolumn{2}{|c|}{8071,95022} & \multicolumn{2}{|c|}{8071,92481} \\
\hline \multirow{4}{*}{500} & 0.01 & \multirow{4}{*}{35} & 9190.50576 & 4.93653 & 9190.50576 & 4.93652 \\
\hline & 0.02 & & 8004.13357 & 4.67673 & 8004.13357 & 4.67673 \\
\hline & 0.03 & & 6663.78341 & 4.37595 & 6663.78341 & 4.37598 \\
\hline & 0.04 & & 5175.71261 & 4.01388 & 5175.71262 & 4.01386 \\
\hline \multicolumn{3}{|c|}{ Slope1 for $R_{a c}=g_{2}\left(\phi_{0}\right)$ and $a_{c}=h_{2}\left(\phi_{0}\right)$} & $-133847,29610$ & $-30,68730$ & $-133847,29580$ & $-30,68730$ \\
\hline \multicolumn{3}{|c|}{ Slope2 for $R_{a c}=f\left(a_{c}\right)$} & \multicolumn{2}{|c|}{4353,65216} & \multicolumn{2}{|c|}{4353,63155} \\
\hline \multirow{4}{*}{700} & \multirow{4}{*}{0.02} & 30 & 12220.32039 & 5.10955 & 12220.32039 & 5.10950 \\
\hline & & 35 & 11231.94926 & 5.05038 & 11231.94926 & 5.05039 \\
\hline & & 40 & 10120.77628 & 4.97275 & 10120.77628 & 4.97275 \\
\hline & & 45 & 8877.84191 & 4.87224 & 8877.84191 & 4.87221 \\
\hline \multicolumn{3}{|c|}{ Slope1 for $R_{a c}=g_{3}\left(d_{n p}\right)$ and $a_{c}=h_{3}\left(d_{n p}\right)$} & $-222,77216$ & $-0,01579$ & $-222,77216$ & $-0,01579$ \\
\hline \multicolumn{3}{|c|}{ Slope2 for $R_{a c}=f\left(a_{c}\right)$} & \multicolumn{2}{|c|}{13999,50057} & \multicolumn{2}{|c|}{13999,73352} \\
\hline
\end{tabular}


Table 5 Comparison of our numerical results with the Chandrasekhar's and Wakif's results, in the case of electrically conducting fluids (i. e. , $\phi_{0}=0$ ).

\begin{tabular}{|c|c|c|c|c|c|c|c|c|}
\hline \multirow{3}{*}{$Q$} & \multicolumn{4}{|c|}{ Existing Literature Results } & \multicolumn{4}{|c|}{ Present Numerical Results } \\
\hline & \multicolumn{2}{|c|}{$\begin{array}{c}\text { VM } \\
\text { (Chandrasekhar, 1961) }\end{array}$} & \multicolumn{2}{|c|}{$\begin{array}{c}\text { RKFM } \\
\text { (Wakif et al., 2018b) }\end{array}$} & \multicolumn{2}{|c|}{$\begin{array}{l}\text { CGLSM } \\
(\mathrm{N}=15)\end{array}$} & \multicolumn{2}{|c|}{$\begin{array}{c}\text { GDQM } \\
(\mathrm{N}=15)\end{array}$} \\
\hline & $R_{a c}$ & $a_{c}$ & $R_{a c}$ & $a_{c}$ & $R_{a c}$ & $a_{c}$ & $R_{a c}$ & $a_{c}$ \\
\hline 0 & 1707.8 & 3.13 & 1707.7617 & 3.1163 & 1707.7617 & 3.1163 & 1707.7617 & 3.1163 \\
\hline 10 & 1945.9 & 3.25 & 1945.7457 & 3.2653 & 1945.7456 & 3.2652 & 1945.7456 & 3.2652 \\
\hline 50 & 2802.1 & 3.68 & 2802.0058 & 3.6792 & 2802.0058 & 3.6792 & 2802.0058 & 3.6792 \\
\hline 100 & 3757.4 & 4.00 & 3757.2301 & 4.0120 & 3757.2301 & 4.0120 & 3757.2301 & 4.0120 \\
\hline 200 & 5488.6 & 4.45 & 5488.5332 & 4.4458 & 5488.5332 & 4.4458 & 5488.5332 & 4.4458 \\
\hline
\end{tabular}

Table 6 Comparison of our numerical and semi-analytical results given by CGLSM, GDQM and WGWRT, in the case of electrically conducting nanofluids, when $R_{N}=0.1, L_{e}=5000, N_{b}=10^{-6}$ and $N_{t}=10^{-6}$.

\begin{tabular}{|c|c|c|c|c|c|c|}
\hline \multirow{3}{*}{$Q$} & \multicolumn{6}{|c|}{ Present Numerical and Semi-Analytical Results } \\
\hline & \multicolumn{2}{|c|}{$\operatorname{CGLSM}(N=28)$} & \multicolumn{2}{|c|}{$\operatorname{GDQM}(N=28)$} & \multicolumn{2}{|c|}{ WGWRT $(N=15)$} \\
\hline & $R_{a c}$ & $a_{c}$ & $R_{a c}$ & $a_{c}$ & $R_{a c}$ & $a_{c}$ \\
\hline 0 & 846.86043 & 2.43637 & 846.86042 & 2.43637 & 846.86043 & 2.43638 \\
\hline 100 & 3091.08440 & 3.83811 & 3091.08440 & 3.83812 & 3091.08440 & 3.83812 \\
\hline 200 & 4860.63528 & 4.33833 & 4860.63528 & 4.33834 & 4860.63528 & 4.33833 \\
\hline 300 & 6485.38722 & 4.66476 & 6485.38722 & 4.66473 & 6485.38722 & 4.66474 \\
\hline 400 & 8029.93455 & 4.91140 & 8029.93455 & 4.91143 & 8029.93455 & 4.91142 \\
\hline 500 & 9521.32407 & 5.11151 & 9521.32407 & 5.11151 & 9521.32407 & 5.11151 \\
\hline 600 & 10974.01001 & 5.28079 & 10974.01001 & 5.28078 & 10974.01001 & 5.28078 \\
\hline 700 & 12396.81589 & 5.42804 & 12396.81589 & 5.42804 & 12396.81589 & 5.42805 \\
\hline 800 & 13795.60743 & 5.55875 & 13795.60743 & 5.55874 & 13795.60744 & 5.55875 \\
\hline 900 & 15174.52413 & 5.67649 & 15174.52414 & 5.67648 & 15174.52414 & 5.67649 \\
\hline 1000 & 16536.61956 & 5.78378 & 16536.61956 & 5.78377 & 16536.61958 & 5.78379 \\
\hline
\end{tabular}

\section{CLOSING REMARKS}

A generalized nanofluid model has been developed in this investigation for alumina-water nanofluids, in order to examine the thermo-magnetohydrodynamic stability in a thin nanofluid layer. Under the OberbeckBoussinesq approximation, the governing partial differential equations (PDEs) were derived formally based on Buongiorno's, Chon's and Corcione's nanofluid models. By applying the linear stability theory and the normal mode analysis method, the resulting dimensionless PDEs are converted into a set of dimensionless ordinary differential equations (ODEs). These ODEs together with the boundary conditions constitute an eigenvalue problem, which has been solved numerically using Chebyshev-Gauss-Lobatto Spectral Method (CGLSM) and Generalized Differential Quadrature Method (GDQM). Moreover, our numerical findings have been validated and discussed in detail via tabular and graphical illustrations, in the case of isothermal impermeable plates with no-slip and zero nanoparticles mass flux conditions. The important findings derived from the present analysis are summarized as follows:

- The numerical results given by CGLSM and GDQM are presented in this paper with an absolute accuracy of the order of $10^{-6}$ and validated with the results of other powerful methods like VM, WGWRT and RKFM.

- Due to the zero nanoparticles mass flux condition, the volumetric fraction of alumina nanoparticles $\mathrm{Al}_{2} \mathrm{O}_{3}$ is controlled passively by the resulting temperature gradient on the impermeable plates.
- $\quad$ Based on the results of the proposed nanofluid model, it is found that the basic dimensional volumetric fraction of alumina nanoparticles $\mathrm{Al}_{2} \mathrm{O}_{3}$ at the hot plate $\phi_{h}^{*}$ and cold plate $\phi_{c}^{*}$ verify the two criterions $\phi_{h}^{*}+\phi_{c}^{*}=2 \phi_{0}$ and $\phi_{c}^{*}>\phi_{h}^{*}$.

- The thermo-magneto-hydrodynamic stability of alumina-water nanofluids increases with the increase in the magnetic Chandrasekhar number $Q$, while it decreases significantly with the increase in the volumetric fraction $\phi_{0}$ and the diameter $d_{n p}$ of alumina nanoparticles.

- $\quad$ The presence of an applied transverse magnetic field has a stabilizing effect on the onset of magneto-convection in alumina-water nanofluids.

- $\quad$ Brownian motion and thermophoresis slip mechanisms have a destabilizing impact on the onset of magneto-convection in alumina-water nanofluids.

- The electrically conducting fluids are generally more stable than their corresponding nanofluids.

- An increase in the magnetic Chandrasekhar number $Q$ leads to a reduction in the critical size of convection cells $L_{c}$, while the volumetric fraction $\phi_{0}$ and the diameter $d_{n p}$ of alumina nanoparticles $\mathrm{Al}_{2} \mathrm{O}_{3}$ increase the critical size of convection cells $L_{c}$. 


\section{ACKNOWLEDGEMENTS}

The authors wish to express their sincere gratitude to the peer reviewers, for their helpful suggestions and valuable comments, which have improved the paper appreciably.

\section{REFERENCES}

Akbarzadeh, P. (2018). "The Onset of MHD Nanofluid Convection Between a Porous Layer in the Presence of Purely Internal Heat Source and Chemical Reaction." Journal of Thermal Analysis and Calorimetry, 131, 2657-2672.

https://doi.org/10.1007/s10973-017-6710-9

Alsabery, A. I., Sheremet, M. A., Chamkha, A. J., and Hashim, I. (2018). "Conjugate Natural Convection of Al2O3-Water Nanofluid in a Square Cavity with a Concentric Solid Insert Using Buongiorno's Two-Phase Model.” International Journal of Mechanical Sciences, 136, 200-219. https://doi.org/10.1016/i.ijmecsci.2017.12.025

Amanulla, C. H., Wakif, A., Boulahia, Z., Suryanarayana Reddy, M., and Nagendra, N. (2018). "Numerical Investigations on Magnetic Field Modeling for Carreau Non-Newtonian Fluid Flow Past an Isothermal Sphere." Journal of the Brazilian Society of Mechanical Sciences and Engineering, 40:462, 1-15.

https://doi.org/10.1007/s40430-018-1385-0

Angayarkanni, S. A., and Philip, J. (2015). "Review on Thermal Properties of Nanofluids : Recent Developments." Advances in Colloid and Interface Science, 225, 146-176.

https://doi.org/10.1016/j.cis.2015.08.014

Animasaun, I. L. (2016) " $47 \mathrm{~nm}$ alumina-water nanofluid flow within boundary layer formed on upper horizontal surface of paraboloid of revolution in the presence of quartic autocatalysis chemical reaction." Alexandria Engineering Journal, 55, 2375 - 2389

http://dx.doi.org/10.1016/j.aej.2016.04.030

Animasaun, I. L., Koriko, O. K., Adegbie, K. S., Babatunde, H. A., Ibraheem, R. O., Sandeep, N., and Mahanthesh, B. (2018). "Comparative analysis between $36 \mathrm{~nm}$ and $47 \mathrm{~nm}$ alumina-water nanofluid flows in the presence of Hall effect." Journal of Thermal Analysis and Calorimetry., $1-14$.

http://dx.doi.org/10.1007/s10973-018-7379-4

Animasaun, I. L. and Pop I. (2017) "Numerical exploration of a nonNewtonian Carreau fluid flow driven by catalytic surface reactions on an upper horizontal surface of a paraboloid of revolution, buoyancy and stretching at the free stream." Alexandria Engineering Journal, 56, 647 658.

https://doi.org/ 10.1016/j.aej.2017.07.005

Astanina, M. S., Abu-Nada, E., and Sheremet, M. A. (2018). "Combined Effects of Thermophoresis, Brownian Motion, and Nanofluid Variable Properties on $\mathrm{CuO}-$ Water Nanofluid Natural Convection in a Partially Heated Square Cavity." Journal of Heat Transfer, 140, 1-12.

https://doi.org/10.1115/1.4039217

Boulahia, Z., Wakif, A., and Sehaqui, R. (2016). "Numerical Study of Mixed Convection of the Nanofluids in Two-Sided Lid-Driven Square Cavity with a Pair of Triangular Heating Cylinders." Journal of Engineering, 2016(Article ID 8962091), 1-8. https://doi.org/10.1155/2016/8962091

Boulahia, Z., Wakif, A., Chamkha, A. J., and Sehaqui, R. (2017a). "Numerical Study of Natural and Mixed Convection in a Square Cavity Filled by a $\mathrm{Cu}$-Water Nanofluid with Circular Heating and Cooling Cylinders." Mechanics and Industry, 18, 1-21.

https://doi.org/10.1051/meca/2017021

Boulahia, Z., Wakif, A., and Sehaqui, R. (2017b). "Finite Volume Analysis of Free Convection Heat Transfer in a Square Enclosure Filled by a $\mathrm{Cu}$-Water Nanofluid Containing Different Shapes of Heating Cylinder." Journal of Nanofluids, 6, 761-768.

https://doi.org/10.1166/jon.2017.1363
Boulahia, Z., Wakif, A., and Sehaqui, R. (2017c). "Modeling of Free Convection Heat Transfer Utilizing Nanofluid Inside a Wavy Enclosure with a Pair of Hot and Cold Cylinders." Frontiers in Heat and Mass Transfer (FHMT), 8, 1-10. https://doi.org/10.5098/hmt.8.14

Boulahia, Z., Wakif, A., and Sehaqui, R. (2017d). "Numerical Modeling of Natural Convection Heat Transfer in a Wavy Wall Enclosure Filled by a $\mathrm{Cu}$ - Water Nanofluid with a Square Cooler." Journal of Nanofluids, $\mathbf{6}$, 324-333.

https://doi.org/10.1166/jon.2017.1315

Boulahia, Z., Wakif, A., and Sehaqui, R. (2018). "Heat Transfer and CuWater Nanofluid Flow in a Ventilated Cavity Having Central Cooling Cylinder and Heated from the Below Considering Three Different Outlet Port Locations." Frontiers in Heat and Mass Transfer, 11, 1-10. https://doi.org/10.5098/hmt.11.11

Buongiorno, J. (2006). "Convective Transport in Nanofluids." Journal of Heat Transfer, 128, 240-250. https://doi.org/10.1115/1.2150834

Buongiorno, J. et al . (2009). "A Benchmark Study on the Thermal Conductivity of Nanofluids." Journal of Applied Physics, 106, 1-14. https://doi.org/10.1063/1.3245330

Canuto, C., Hussaini, M. Y., Quarteroni, A. M., and Thomas Jr, A. (2012). Spectral Methods in Fluid Dynamics. Springer Science \& Business Media.

Chandrasekhar, S. (1961). Hydrodynamic and hydromagnetic stability. Oxford University Press, Oxford.

Choi, S. U. S. (1995). "Enhancing Tthermal Conductivity of Fluids with Nanoparticles." ASME - Publications - Fed, 231, 99-106.

Chon, C. H., Kihm, K. D., Lee, S. P., and Choi, S. U. S. (2005). "Empirical Correlation Finding the Role of Temperature and Particle Size for Nanofluid (A12O3) Thermal Conductivity Enhancement." Applied Physics Letters, 87, 1-3.

https://doi.org/10.1063/1.2093936

Corcione, M. (2011). "Empirical Correlating Equations for Predicting the Effective Thermal Conductivity and Dynamic Viscosity of Nanofluids." Energy Conversion and Management, 52, 789-793.

https://doi.org/10.1016/j.enconman.2010.06.072

Fidanoglu, M., Baskaya, E., Komurgoz, G., and Ozkol, I. (2014). "Application of Differential Quadrature Method and Evolutionary Algorithm to MHD Fully Developed Flow of a Couple-Stress Fluid in a Vertical Channel With Viscous Dissipation and Oscillating Wall Temperature." ASME 2014 12th Biennial Conference on Engineering Systems Design and Analysis, 1-9. https://doi.org/10.1115/ESDA2014-20137

Garnett, J. C. M. (1905). "Colours in Metal Glasses, in Metallic Films and in Metallic Solutions." Proc. R. Soc. Lond. A, 76, 370-373.

Garoosi, F., and Talebi, F. (2017). "Numerical Simulation of Conjugate Conduction and Natural Convection Heat Transfer of Nanofluid Inside a Square Enclosure Containing a Conductive Partition and Several Disconnected Conducting Solid Blocks Using the Buongiorno's Two Phase Model." Powder Technology, 317, 48-71. https://doi.org/10.1016/j.powtec.2017.04.042

Koriko, O. K., Animasaun, I. L., Mahanthesh, B., Saleem, S., Sarojamma, G., and Sivaraj, R. (2018). "Heat Transfer in the Flow of Blood-gold Carreau Nanofluid Induced by Partial Slip and Buoyancy." Heat Transfer-Asian Research, 47, 806-823. https://doi.org/10.1002/htj.21342

Makinde, O. D. , and Animasaun, I. L. (2016a). "Bioconvection in MHD nanofluid flow with nonlinear thermal radiation and quartic autocatalysis chemical reaction past an upper surface of a paraboloid of revolution." International Journal of Thermal Sciences, 109, 159 - 171. 14 https://doi.org/10.1016/j.ijthermalsci.2016.06.003 
Makinde, O. D. , and Animasaun, I. L. (2016b). "Thermophoresis and Brownian motion effects on MHD bioconvection of nanofluid with nonlinear thermal radiation and quartic chemical reaction past an upper horizontal surface of a paraboloid of revolution." Journal of Molecular Liquids, 221, 733 - 743.

Makinde, O.D., Omojola, M.T., Mahanthesh, B., Alao, F.I., Adegbie, K.S., Animasaun, I. L., Wakif , A., Sivaraj, R., and Tshehla, M.S. (2018). "Significance of Buoyancy, Velocity Index and Thickness of an Upper Horizontal Surface of a Paraboloid of Revolution: The Case of NonNewtonian Carreau Fluid." Defect and Diffusion Forum, 387, 550-561. https://doi.org/10.4028/www.scientific.net/DDF.387.550

Maxwell, J.C.A., (1873). Treatise on electricity and magnetism. 2nd ed. Oxford, UK: Clarendon Press.

https://doi.org/10.1016/j.molliq.2016.06.047

McNab, G. S., and Meisen, A. (1973). "Thermophoresis in Liquids." Journal of Colloid and Interface Science, 44, 339-346.

Mehmood, K., Hussain, S., and Sagheer, M. (2017). "Mixed Convection in Alumina-Water Nanofluid Filled Lid-Driven Square Cavity with an Isothermally Heated Square Blockage Inside with Magnetic Field Effect : Introduction." International Journal of Heat and Mass Transfer, 109, 397-409.

https://doi.org/10.1016/j.ijheatmasstransfer.2017.01.117

Naddaf, A., and Heris, S. Z. (2018). "Experimental Study on Thermal Conductivity and Electrical Conductivity of Diesel Oil-Based Nanofluids of Graphene Nanoplatelets and Carbon Nanotubes." International Communications in Heat and Mass Transfer, 95, 116-122.

https://doi.org/10.1016/j.icheatmasstransfer.2018.05.004

Nguyen, C. T., Desgranges, F., Roy, G., Galanis, N., Mare, T., Boucher, S., and Minsta, H. A. (2007). "Temperature and Particle-Size Dependent Viscosity Data for Water Based Nanofluids- Hysteresis Phenomenon." Int. J. Heat Fluid Flow, 28, 1492-1506.

https://doi.org/10.1016/j.ijheatfluidflow.2007.02.004

Nield, D. A., and Kuznetsov, A. V. (2014a). "The Onset of Convection in a Horizontal Nanofluid Layer of Finite Depth : A Revised Model." International Journal of Heat and Mass Transfer, 77, 915-918.

https://doi.org/10.1016/j.ijheatmasstransfer.2014.06.020

Nield, D. A., and Kuznetsov, A. V. (2014b). "Thermal Instability in a Porous Medium Layer Saturated by a Nanofluid: A Revised Model." International Journal of Heat and Mass Transfer, 68, 211-214.

https://doi.org/10.1016/j.ijheatmasstransfer.2013.09.026

Pandey, A. K., and Kumar, M. (2016). "Effect of Viscous Dissipation and Suction/Injection on MHD Nanofluid Flow Over a Wedge with Porous Medium and Slip." Alexandria Engineering Journal, 55, 3115-3123.

https://doi.org/10.1016/j.aej.2016.08.018

Pandey, A. K., and Kumar, M. (2017a). "Boundary Layer Flow and Heat Transfer Analysis on Cu-Water Nanofluid Flow Over a Stretching Cylinder with Slip.” Alexandria Engineering Journal, 56, 671-677.

https://doi.org/10.1016/j.aej.2017.01.017

Pandey, A. K., and Kumar, M. (2017b). "Chemical Reaction and Thermal Radiation Effects on Boundary Layer Flow of Nanofluid Over a Wedge with Viscous and Ohmic Dissipation." St. Petersburg Polytechnical University Journal: Physics and Mathematics, 3, 322-332.

https://doi.org/https://doi.org/10.1016/j.spjpm.2017.10.008

Pandey, A. K., and Kumar, M. (2017c). "Natural Convection and Thermal Radiation Influence on Nanofluid Flow Over a Stretching Cylinder in a Porous Medium with Viscous Dissipation." Alexandria Engineering Journal, 56, 55-62.

https://doi.org/10.1016/j.aej.2016.08.035

Qasim, M., Ali, Z., Wakif, A., and Boulahia, Z. (2018). 'Numerical Simulation of MHD Peristaltic Flow with Variable Electrical Conductivity and Joule Dissipation Using Generalized Differential Quadrature Method." Communications in Theoretical Physics, In Press.
Rana, P., Khurana, M., and Srivastava, S. (2017). "Linear Stability Analysis on the Onset of MHD Non-Newtonian Viscoelastic Rotating Nanofluid Layer with Heat Generation." In AIP Conference Proceedings (Vol. 1897, pp. 1-8). AIP Publishing.

https://doi.org/10.1063/1.5008709

Schenck, J. F. (1996). "The Role of Magnetic Susceptibility in Magnetic Resonance Imaging: MRI Magnetic Compatibility of the First and Second Kinds." Medical Physics, 23, 815-850. https://doi.org/10.1118/1.597854

Shah, N. A., Animasaun, I. L., Ibraheem, R. O., Babatunde, H. A., Sandeep, N., and Pop, I. (2018). "Scrutinization of the Effects of Grashof Number on the Flow of Different Fluids Driven by Convection over Various Surfaces." Journal of Molecular Liquids, 249, 980-990. https://doi.org/10.1016/j.molliq.2017.11.042

Shu, C. (2012). Differential quadrature and its application in engineering. Springer Science \& Business Media.

Sihvola, A. H., and Lindell, I. V. (1992). "Effective Permeability of Mixtures.” Progr. Electromagn. Res., 6, 153-180.

Trefethen, L. N. (2000). Spectral Methods in MATLAB. SIAM.

Wakif, A., Boulahia, Z., Ali, F., Eid, M. R., and Sehaqui, R. (2018a). "Numerical Analysis of the Unsteady Natural Convection MHD Couette Nanofluid Flow in the Presence of Thermal Radiation Using Single and Two-Phase Nanofluid Models for $\mathrm{Cu}$-Water Nanofluids." International Journal of Applied and Computational Mathematics, 4 : 81, 1-27. https://doi.org/10.1007/s40819-018-0513-y

Wakif, A., Boulahia, Z., and Sehaqui, R. (2017a). "Numerical Analysis of the Onset of Longitudinal Convective Rolls in a Porous Medium Saturated by an Electrically Conducting Nanofluid in the Presence of an External Magnetic Field." Results in Physics, 7, 2134-2152.

https://doi.org/10.1016/j.rinp.2017.06.003

Wakif, A., Boulahia, Z., Mishra, S. R., Rashidi, M. M., and Sehaqui, R. (2018b). "Influence of a Uniform Transverse Magnetic Field on the Thermo - Hydrodynamic Stability in Water-Based Nanofluids with Metallic Nanoparticles Using the Generalized Buongiorno's Mathematical Model." The European Physical Journal Plus, 133:181, 116.

https://doi.org/10.1140/epjp/i2018-12037-7

Wakif, A., Boulahia, Z., and Sehaqui, R. (2018c). "A Semi-Analytical Analysis of Electro-Thermo-Hydrodynamic Stability in Dielectric Nanofluids Using Buongiorno's Mathematical Model Together with More Realistic Boundary Conditions." Results in Physics, 9, 1438-1454. https://doi.org/10.1016/j.rinp.2018.01.066

Wakif, A., Boulahia, Z., and Sehaqui, R. (2017b). "Numerical Study of the Onset of Convection in a Newtonian Nanofluid Layer with Spatially Uniform and Non Uniform Internal Heating." Journal of Nanofluids, 6, 136-148.

https://doi.org/10.1166/jon.2017.1293

Wakif, A., Boulahia, Z., and Sehaqui, R. (2016). "Analytical and Numerical Study of the Onset of Electroconvection in a Dielectric Nanofluid Saturated a Rotating Darcy Porous Medium." International Journal of Advanced Computer Science and Applications, 7, 299-311. https://doi.org/10.14569/ijacsa.2016.070841

Żyła, G., and Fal, J. (2017). "Viscosity, Thermal and Electrical Conductivity of Silicon Dioxide-Ethylene Glycol Transparent Nanofluids: An Experimental Studies." Thermochimica Acta, 650, 106113.

https://doi.org/10.1016/j.tca.2017.02.001

Żyła, G., Vallejo, J. P., Fal, J., and Lugo, L. (2018). "NanodiamondsEthylene Glycol Nanofluids: Experimental Investigation of Fundamental Physical Properties." International Journal of Heat and Mass Transfer, 121, 1201-1213.

https://doi.org/10.1016/j.ijheatmasstransfer.2018.01.073 